\title{
The AGASA/SUGAR Anisotropies and TeV Gamma Rays from the Galactic Center: A Possible Signature of Extremely High-energy Neutrons
}

\author{
Roland M. Crocker ${ }^{1,2}$, Marco Fatuzzo ${ }^{3}$, Randy Jokipii ${ }^{4}$, Fulvio Melia ${ }^{5}$ \\ and Raymond R. Volkas ${ }^{2}$ \\ ${ }^{1}$ Harvard-Smithsonian Center for Astrophysics \\ 60 Garden St., Cambridge MA 02138 \\ rcrocker@cfa.harvard.edu \\ ${ }^{2}$ Research Centre for High Energy Physics, School of Physics, \\ The University of Melbourne, 3010 Australia \\ r.crocker,r.volkas@physics.unimelb.edu.au \\ ${ }^{3}$ Physics Department, \\ Xavier University, Cincinnati, $\mathrm{OH} 45207$ \\ fatuzzo@cerebro.cs.xu.edu \\ ${ }^{4}$ Department of Planetary Sciences, \\ The University of Arizona, Tucson, AZ 85721 \\ jokipii@lpl.arizona.edu \\ ${ }^{5}$ Physics Department and Steward Observatory, \\ The University of Arizona, Tucson, AZ 85721 \\ melia@physics.arizona.edu
}

\begin{abstract}
Recent analysis of data sets from two extensive air shower cosmic ray detectors shows tantalizing evidence of an anisotropic overabundance of cosmic rays towards the Galactic Center (GC) that "turns on" around $10^{18} \mathrm{eV}$. We demonstrate that the anisotropy could be due to neutrons created at the Galactic Center through charge-exchange in proton-proton collisions, where the incident, high energy protons obey an $\sim E^{-2}$ power law associated with acceleration at a strong shock. We show that the normalization supplied by the gamma-ray signal from EGRET GC source 3EG J1746-2851 - ascribed to pp induced neutral pion decay at $\mathrm{GeV}$ energies - together with a very reasonable spectral index of 2.2, predicts a neutron flux at $\sim 10^{18} \mathrm{eV}$ fully consistent with the extremely high energy cosmic
\end{abstract}


ray data. Likewise, the normalization supplied by the very recent GC data from the HESS air-Cerenkov telescope at TeV energies is almost equally-well compatible with the $\sim 10^{18} \mathrm{eV}$ cosmic ray data. Interestingly, however, the EGRET and HESS data appear to be themselves incompatible. We consider the implications of this discrepancy. We discuss why the Galactic Center environment can allow diffusive shock acceleration at strong shocks up to energies approaching the ankle in the cosmic ray spectrum. Finally, we argue that the shock acceleration may be occuring in the shell of Sagittarius A East, an unusual supernova remnant located very close to the Galactic Center. If this connection between the anisotropy and Sagittarius A East could be firmly established it would be the first direct evidence for a particular Galactic source of cosmic rays up to energies near the ankle.

Subject headings: acceleration of particles — cosmic-rays — radiation mechanisms:nonthermal — supernova remnants

\section{Introduction}

The origin of cosmic rays $(\mathrm{CRs})$ is one of the most important unsolved problems in astrophysics. While it has long been speculated that diffusive shock acceleration of protons and ions at shock fronts associated with supernova remnants (SNRs) is the mechanism likely responsible for accelerating the bulk of high energy cosmic rays, definitive observational proof has been elusive. Further, the conditions at almost all known SNRs seem not to promote the acceleration of CRs beyond the 'knee' feature in the spectrum at $\simeq 5 \times 10^{15} \mathrm{eV}$. The acceleration mechanism for CRs between the knee and the 'ankle' at few $\times 10^{19} \mathrm{eV}$ therefore seems to be an even deeper puzzle.

The purpose of this paper is to argue that the Galactic Center (GC), a region with relatively extreme conditions compared to the rest of the Milky Way, is a likely site where CRs are accelerated up to the ankle. Our argument is based on the following: (i) The EGRET $\gamma$-ray source 3EG J1746-2851, most likely located near the GC, provides good evidence for pion production from high energy proton-proton ( $\mathrm{pp}$ ) collisions. Neutrons will then inevitably also be produced by this source. (ii) The $\mathrm{TeV} \gamma$-rays from the direction of the GC detected by a number of air Cerenkov telescopes, in particular, the HESS instrument, also support the notion that high-energy proton acceleration and collision processes (again, leading inevitably to neutron production) are occuring in this region. (iii) The AGASA CR anisotropy for the energy range $10^{17.9}-10^{18.5} \mathrm{eV}$ is consistent with high energy neutron emission from the GC. (iv) The reanalysed SUGAR data also reveal an anisotropy for this 
energy regime from a direction close to the GC. (v) The SNR Sgr A East provides a plausible specific GC system where hadronic acceleration up to the ankle can occur, differing as it does from other Galactic SNRs by the special conditions of its GC environment.

If the connection between the anisotropies and Sgr A East could be firmly established, it would be the first identification of a specific source producing high energy, Galactic CRs, and, moreover, it would be proven to be an important, possibly unique, contributor to CR acceleration up to the ankle. The southern-hemisphere AUGER detector, currently under construction, will test our hypothesis in the relatively near future: it should see a significant point source of $\sim 10^{18} \mathrm{eV}$ neutrons at the GC. (For lower energies, previous work has shown that a GC neutrino signal should also be seen by a northern-hemisphere $\mathrm{km}^{3}$ neutrino telescope: Crocker et al. 2002).

We extend earlier work on GC CR production in three important ways. First, postulating a GC source of high energy protons obeying an $\sim E^{-2}$ power law at the source, with normalisation fixed by the concomitant $(\mathrm{GeV})$ EGRET $\gamma$-ray observations of 3EG J1746-2851, we calculate neutron production through charge-exchange $p p \rightarrow n X$ reactions. Quite non-trivially, we find neutron fluxes consistent with the magnitudes of the AGASA and $S U G A R$ anisotropies. Second, we likewise show that a simultaneous fit to the (TeV) data supplied by the HESS instrument and the cosmic ray anisotropy data is also consistent with the existence of a population of of shock-accelerated protons governed by a $\sim E^{-2}$ power law up to extremely high energies. Third, we show that diffusive shock acceleration beyond the knee can occur provided that there is a significant magnetic field component perpendicular to the SNR shock propagation direction (i.e., parallel to the shock front). We explain why the special conditions at the GC, especially the higher density of the ambient medium and higher magnetic field, can realise this situation. Third, we argue that the specific GC SNR Sgr A East is the most likely specific candidate site for CR acceleration up to the ankle. Note, however, that it is not necessary to identify the specific source for the conclusion italicised above to follow.

Note also that we admit from the start that our model is not consistent with all available data. The data, however, are inconsistent amongst themselves in the two important instances where there is disagreement with our model (see $\S 3.3$ and $\S 5.4$ ). These instances of disagreement - both discussed in further detail below - are (i) that the SUGAR results indicate a point source offset by $7.5^{\circ}$ from the GC (whereas we predict, of course, a source at the position of the GC on the sky) and (ii) that the $\sim \mathrm{TeV}$ measurements of the $\gamma$-ray flux from the GC (by a number of instruments) appears to be deficient compared to what we would expect. We discuss a number of possible resolutions of this discrepancy, finding the most favorable to hinge on there being two effective GC sources of $\gamma$-rays, an idea which 
has some support from current observations.

\section{Origin of Cosmic Rays: Role of Neutrons}

The hypothesis that shocks at SNRs are responsible for the acceleration of CRs over the bulk of the observed spectrum is fifty years old (Shklovskii 1953). And, indeed, there is strong, albeit circumstantial, evidence that SNRs do accelerate cosmic rays up to, at least, the 'knee' in the spectrum at a few $\mathrm{PeV}$ (we set $E_{\text {knee }} \equiv 10^{15} \mathrm{eV}$ for definiteness). This evidence comes primarily from two arguments, viz,

1. Supernovae seem to be the only class of Galactic object able to inject the power necessary to maintain the observed cosmic ray output of about $10^{48} \mathrm{ergs} /$ year (e.g., Longair (1994)).

2. The cosmic ray spectrum is governed by a power law with spectral index around 2.7. This is close to the universal power law of spectral index $\sim 2$ theoretically expected from diffusive shock acceleration at the sort of strong shock associated with a supernova blast wave. (Note that this theoretical expectation for spectral indices close to two - at the source - is observationally confirmed by radio and $\gamma$-ray data from various SNRs.) The difference between these two indices, further, can be compellingly explained as arising from energy-dependent propagation/confinement effects.

However, there is yet no direct observational evidence for the SNR-CR connection and certainly no particular SNR has been proven to be a CR source. Further, many researchers have found that their models are pushed to accelerate particles up to $E_{\text {knee }}$. This becomes doubly troubling given the fact that, as emphasized by Jokipii and Morfill (1991), matching the spectra at the knee requires, short of a cosmic conspiracy, that the population of cosmic rays above the knee is closely related to that below the knee. In fact, there are good reasons to think that the bulk of the cosmic rays are Galactic in origin up to the 'ankle' in the spectrum at a few $\times 10 \mathrm{EeV}\left(1 \mathrm{EeV} \equiv 10^{18} \mathrm{eV}\right)$, not the least of which is that a proton of this energy has a gyroradius the size of the radius of the Galactic disk. ${ }^{1}$

We would like, therefore, to determine whether it is, indeed, the case that SNRs can accelerate particles up to energies of $10^{18-19} \mathrm{eV}$. Further, it would be helpful to find evidence

\footnotetext{
${ }^{1}$ Yet higher energy CRs may therefore no longer be confined to our Galaxy, so are almost certainly extraGalactic. The up-turn in the spectrum at the ankle is nicely consistent with a new population taking over from a diminishing Galactic component.
} 
that some particular object is accelerating particles to these extremely high energies (EHEs).

We obviously need to look for a signal in electrically neutral particles, so that the location of the source is not scrambled by deflection due to the Galactic magnetic field. ${ }^{2}$ There are three main candidates: photons, neutrinos and neutrons. Photons with energy $10^{17,18} \mathrm{eV}$ will be produced by the decay of neutral pions produced by hadronic collisions. However, such EHE $\gamma$ 's will interact strongly with background media long before reaching the Earth, so they are not useful as direct probes at those energies. The flux of $10^{17,18} \mathrm{eV}$ neutrinos, another concomitant of hadronic collisions, is expected to be orders of magnitude too small to be seen by future $\mathrm{km}^{3}$ neutrino telescopes. Although three of us have previously shown that the GC should emit neutrinos (Crocker et al. 2000; Crocker et al. 2002) [see also (Alvarez-Muñiz and Halzen 2002)], their detection could not settle the question of where the $10^{18} \mathrm{eV}$ CRs are coming from, because the detectable flux of neutrinos lies at considerably lower energies.

This leaves us with neutrons. As for neutrinos and $\gamma$-rays, neutrons are an inescapable concomitant of hadronic acceleration of protons and ions: charge exchange occurs in a nonnegligible fraction of all interactions between accelerated protons and ambient protons. Neutrons are also produced in $\mathrm{p} \gamma$ collisions and dissociations of accelerated ions (see below). The neutron, however, is unstable in free space, a fact we have to take into account when contemplating neutron astronomy.

The neutron is the longest-lived unstable 'elementary' particle with a decay time at rest, $\tau_{n}$, of 886 seconds (Hagiwara and al. 2002). This means that a neutron will travel, on average, a distance of

$$
d_{n}\left(E_{n}\right)=c \gamma_{n} \tau_{n} \simeq 9\left(\frac{E_{n}}{\mathrm{EeV}}\right) \mathrm{kpc},
$$

(where the Lorentz factor is given by $\gamma_{n} \equiv E_{n} / m_{n}$ ) in free space before decaying. What plausibly neutron-producing regions lie within the $\sim 9 \mathrm{kpc}$ distance travelled on average by an EeV neutron? The Galactic Center - one of the most energetic regions in the Galaxy at a distance of around $8.5 \mathrm{kpc}$ is the principal candidate.

What would be a smoking gun signature for EHE CR neutrons? It is in extensive airshowers (EAS) apparently initiated by particles coming from the direction of the GC that one would need to look for GC neutrons. The signal might be very difficult to disentangle, essentially because any neutron-initiated EAS at these energies is likely to be indistinguish-

\footnotetext{
${ }^{2}$ Charged particles can still be used for very close sources. See, for example, Chilingarian (2003) for a study of putative CR emission from the 300 pc distant Monogem ring SNR.
} 
able from a background, proton-initiated $\mathrm{EAS}^{3}$ (which proton, given the presence of the Galactic magnetic field, might originate from a source considerably away from the GC). The best evidence for GC neutrons one might hope for, then, is an anisotropy in the EeV cosmic ray data in the form of an excess towards the GC. Intriguingly, there is tantalizing evidence, which we now briefly review, from two different data sets that just such an anisotropy exists.

\section{The Galactic Center Anisotropy Explained by a GC Source of Protons/Neutrons}

Recent analysis of data from two different cosmic ray detectors has revealed the presence of an anisotropic overabundance of cosmic rays coming from the general direction of the Galactic Center. Consistent with these findings, analysis of data from a third array has found a broad anisotropy along the Galactic plane. We now briefly review each of these findings.

Statistically, the most robust determination for an anisotropy has been by the Akeno Giant Shower Array (AGASA) Group (Hayashida et al. 1999a) which, in analysis of 114,000 airshowers found a strong - 4\% amplitude - anisotropy in the energy range $10^{17.9}-10^{18.3} \mathrm{eV}$ (we label the $10^{17.9} \mathrm{eV}$ energy at which the anisotropy apparently 'turns on' $E_{\text {onset }}$ ). Note that the AGASA Collaboration (Takeda 1999) has estimated that the systematic uncertainty in its instrument's energy callibration is $30 \%$ and we shall adopt this figure in our analysis. The group's two-dimensional analysis of the data showed that this anisotropy could be interpreted as an excess of air showers from two regions each of $\sim 20^{\circ}$ extent, one of $4 \sigma$ significance near the GC and another of $3 \sigma$ in Cygnus. Subsequent re-analysis by the AGASA group incorporating new data - has only served to bolster the claim that the anisotropy is real (Hayashida and al. 1999b) with, this time, a $4.5 \sigma$ excess seen near the GC over a beam size of $20^{\circ}$ between $10^{18.0}-10^{18.4} \mathrm{eV}$.

Prompted by the AGASA result, Bellido et al. (2001) re-analyzed the data collected by the SUGAR cosmic ray detector which operated from 1968 to 1979 near Sydney. Setting a priori an energy range similar to that determined for the AGASA anisotropy, these researchers also found an anisotropy, consistent with a point source located at $7.5^{\circ}$ from the

\footnotetext{
${ }^{3}$ Though note here that there is a theoretical possibility, at least, that empirical data may one day be able to directly distinguish EHE cosmic ray neutrons from protons via characteristic differences between the $\mu^{+}$to $\mu^{-}$ratios seen in the extensive air showers generated by these particles (a proton will produce an excess of $\pi^{+}$and, therefore, $\mu^{+}$in the forward region whereas a neutron will produce an excess of $\pi^{-}$and, therefore, $\mu^{-}$).
} 
$\mathrm{GC}$ - and $6^{\circ}$ degrees from the AGASA maximum over an energy range of $10^{17.9}-10^{18.5} \mathrm{eV}$.

Lastly, the HiRes Collaboration has seen a Galactic Plane enhancement in cosmic ray events in the energy range between $10^{17.3}$ and $10^{18.5} \mathrm{eV}$ with $3.2 \sigma$ confidence (Bird et al. 1999) (this is consistent with the AGASA and SUGAR results because the HiRes study was broad scale and did not attempt to pin down whether any particular Galactic longitudes were responsible for the detected excess: Bellido et al. 2001).

As we shortly set out, the anisotropies mentioned above have what we believe is a natural explanation in terms of neutron emission from the GC region (see the subsection on 'GC Neutron Models' below). Before we discuss this idea in detail, however, we also consider the possibility that the observed anisotropies can be explained directly by a diffusive flux of charged particles from the GC. We label such scenarios 'GC Charged Particle Models'.

\subsection{GC Charged Particle Models}

There has been a concerted effort to model the propagation of charged particles from an assumed source in the vicinity of the GC, through various assumed configurations of the Galactic magnetic field, to Earth to see whether these models can reproduce the observed anisotropies (Clay 2000;Bednarek, Giller, and Zielinska 2002;Candia, Mollerach, and Roulet 2002). In general, researchers have found that a fair degree of correspondence between models and reality can be achieved, with, in particular, an anisotropy becoming evident for $\mathcal{O}[\mathrm{EeV}]$ protons propagating through various field configurations with a magnetic field amplitude of $\mathcal{O}[\mu \mathrm{G}]$. Finding an exact correspondence for 'turn-on' and 'turn-off' energies seems, however, to require quite some fine-tuning of the particulars of $\mathbf{B}_{\text {Galactic }}$ and is not such a robust solution as the neutron idea. Further, for reasonable amplitudes of $\mathbf{B}_{\text {Galactic }}$ modeling studies show that the actual separation seen between the GC and the observed excess will exceed the $\mathcal{O}\left[10^{\circ}\right]$ observed (Candia, Epele, and Roulet 2002). In fact, MedinaTanco and Watson (2001) report that the source must be within $\sim 2 \mathrm{kpc}$ of the Earth to reproduce the observed deflection. This is of course, a possibility, but then the near alignment with the anisotropies and the GC becomes, essentially, a coincidence. Lastly, and perhaps most tellingly, the (consistent with-) point-like anisotropy seen in the SUGAR data is very difficult to reproduce in modeling of charged particle trajectories because the particles tend to smear out until rather higher energies (for reasonable magnetic fields) than $E_{\text {onset }}$. 


\subsection{GC Neutron Models}

The broad idea that neutron emission from the GC may produce an anisotropy was, to our knowledge, first mooted by Jones (1990). The neutron idea was subsequently revived by the AGASA group in the paper announcing their discovery of the GC anisotropy (Hayashida et al. 1999a). The AGASA paper authors pointed out that the anisotropy 'turn on' at a definite energy of $\sim$ EeV finds a natural explanation in the fact that - as outlined above - this energy corresponds to a gamma factor for neutrons large enough that they can reach us from the GC. So, broadly, neutrons below this energy decay in propagation and are then diverted by Galactic magnetic fields. The cessation of the anisotropy above $\sim 10^{18.4}$ $\mathrm{eV}$ can be explained as either due to a very steep GC source spectrum or an actual cut-off in the source so that the background takes over again at this energy.

Most recently, the broad idea above has been considerably refined in the work of Bossa et al. (2003), whose basic scenario we follow and now explain. These authors have made detailed propagation calculations following the trajectories of protons from neutrons that decay in flight from the GC or, to be precise, they follow the trajectories of anti-protons leaving the Earth and calculate the probability that a decay should occur over the interval during which the anti-proton's path points back to the assumed GC source. Using this procedure they calculate detailed maps of the arrival direction of the combined neutron and proton signal for various values of an assumed Galactic magnetic field which has both a regular and a turbulent component. The Bossa et al. (2003) scenario extends that presented by Medina-Tanco and Watson (2001) in which, essentially, decay protons produced close to the Earth arrive from directions close to the GC whereas those produced in the inner Galaxy arrive preferentially from the directions of the spiral arms - thus also neatly explaining the Cygnus anisotropy - as their trajectories wind around the regular magnetic field lines (Bossa et al. 2003).

Crucially, the GC, with declination $\delta=-28.9^{\circ}$, is outside the field of view of AGASA (which is limited to $\delta>-24^{\circ}$; Bossa et al. 2003). This provides a natural explanation for the 'turn off' of the AGASA anisotropy without the need for a source cut-off: at energies $\gtrsim 10^{18.4} \mathrm{eV}$, neutrons do not (on average) decay in flight from the GC, but, instead, travel in a straight line from the GC to Earth to produce a point-like anisotropy out of the field of AGASA. Moreover, Bossa et al. (2003) were also able to reproduce the sharp onset of the AGASA anisotropy at $10^{17.9} \mathrm{eV}$ by positing a Galactic magnetic field random component of fairly large amplitude $(3 \mu \mathrm{G})$ and also assuming a GC source governed by a spectral index of 2.2. As mentioned above, these researchers also relate the Cygnus region excess seen by AGASA to the GC source with a Galactic magnetic field whose regular component is along the spiral arms (and not just azimuthal). Finally, in the Bossa et al. (2003) 
scenario, the magnitudes and morphologies of the AGASA and SUGAR results were shown to be compatible (directional consistency being problematic - see below). In addition to approximate consistency in magnitude, the fact that the AGASA and SUGAR anisotropies are, respectively, diffuse and (consistent with) point-like finds a natural explanation. Indeed, from the requirement that the source normalization generate the observed $4 \%$ amplitude anisotropy of the right-ascension first harmonic (and continuing to assume a power-law source spectrum with index 2.2), Bossa et al. (2003) determine that the total luminosity of the GC source over the specified decade in energy be

$$
L_{G C}\left(10^{17.5}-10^{18.5}\right) \simeq 4 \times 10^{36} \mathrm{erg} \mathrm{s}^{-1},
$$

which implies a direct neutron flux between $10^{17.9}-10^{18.5} \mathrm{eV}$ of $2 \times 10^{-17} \mathrm{~cm}^{-2} \mathrm{~s}^{-1}$ (no error range given), which is not very different from the SUGAR result of $(9 \pm 3) \times 10^{-18} \mathrm{~cm}^{-2} \mathrm{~s}^{-1}$. Bossa et al. (2003) also point out that these two figures need not be exactly equal: protons will be, in general, delayed by many thousands of years with respect to the neutron arrival times so the source intensity need not be exactly the same when the neutrons and protons we observe today were separately emitted.

A couple of important points one should note about the Bossa et al. (2003) scenario (as these researchers themselves remark) are that (i) for the magnetic field adopted, the phase $\left(\sim 330^{\circ}\right)$ of the first harmonic in right ascension found is somewhat larger than that detected by AGASA and (ii) because Bossa et al. (2003) do relate the Cygnus excess to a GC source, this excess should disappear for $E \gtrsim 2 \mathrm{EeV}$ independently of the intrinsic source energy cutoff because any reasonable Galactic magnetic field could not, reasonably, shepherd cosmic rays above this energy along a spiral arm.

\subsection{Non-coincidence of the SUGAR ansisotropy with the GC}

That the AGASA ansisotropy is not exactly coincident with the GC finds a reasonable explanation in the fact already presented that the actual GC is out of the field of view of this instrument. That the SUGAR ansisotropy, however, is not coincident with the GC presents a challenge to all scenarios that would posit that the source of the EHE cosmic rays is at the GC. Either, then, all such scenarios are incorrect or the SUGAR directional determination is somewhat in error. If not the GC, then the SUGAR anisotropy could be due to the supernova remnant W28, which has also been detected by the EGRET instrument in gamma rays. However, W28 (located at $\alpha=274^{\circ}, \delta=-23.18^{\circ}$ ) is itself displaced from the SUGAR position by about $4^{\circ}$, and one would need to again invoke an error in SUGAR's directional determination. For reasons we immediately explain, such an error seems to be a viable possibility for, if the SUGAR directional determination is correct then 
1. in the case that the anisotropy is due to neutrons there must be a completely unknown source at the position suggested by the SUGAR data (unobserved, e.g., by the EGRET instrument in gamma rays)

2. or, alternatively, in the case that the anisotropy is due to protons directly either

(a) there is a source located very close to us which is contrained to be in close but completely coincidental alignment with the direction towards the GC or

(b) there is a source somewhat further away, the particles produced by which - also completely coincidentally - happen to be bent in flight in exactly such a way as to appear to come from close to the direction of the GC (and, further, remain sufficently bunched that their signal is consistent with point-like for SUGAR).

We note, furthermore, that whereas, as stressed above, the GC is outside the field of view of AGASA, the position of the SUGAR maximum is inside the AGASA field of view so that the putative SUGAR source should be seen by AGASA. That it is not means that these two instruments are in disagreement. AGASA, moreover, has the better statistics. We believe, then, that a quite natural reading of the situation is that SUGAR's directional determination is in error. The only other way out of this apparent dilemma is to posit significant variability (between the SUGAR and AGASA observation times) at the source which, however, would in fact be an argument in favor of a point source, rather than diffuse emission.

\section{Evidence for Hadronic Acceleration at the Galactic Center}

Direct evidence of hadronic acceleration at the Galactic center comes from the EGRET detection of a $30 \mathrm{MeV}$ - $10 \mathrm{GeV}$ continuum source (3EG J1746-2851 in the third EGRET catalog: Hartman et al. (1999)) within $1^{\circ}$ of the nucleus (Mayer-Hasselwander et al. 1998) ${ }^{4}$. The EGRET spectrum exhibits a clear break at $\sim 1 \mathrm{GeV}$, and therefore cannot be fit by a single power law. Instead, this break appears to be the signature of a process involving pion decays. Specifically, the decay of neutral pions generated via pp scatterings between relativistic and ambient protons produces a broad $\gamma$-ray feature that mirrors all but the lowest energy

\footnotetext{
${ }^{4}$ The IBIS telescope on board INTEGRAL has also recently released a preliminary result for the detection of a GC source in the EGRET energy range: Di Cocco et al. (2004)
} 
EGRET data. Of course, pp scatterings also produce charged pions which, in turn, decay into "secondary" electrons and positrons. These leptons are capable of producing their own $\gamma$-ray emission via bremsstrahlung and Compton scattering. Interestingly, if the secondary leptons build up to a steady-state distribution balanced by bremsstrahlung and Coulomb losses, the former accounts naturally for the lowest energy EGRET datum, independent of the ambient proton number density. This crucial feature results from the fact that the secondary leptons produce a steady state distribution whose normalization scales as the inverse of the ambient proton number density, whereas the bremsstrahlung emissivity per lepton scales directly with this density. The pion decays link the lepton and photon generation rates, so the bremsstrahlung and pion-decay photon emissivities are tightly correlated.

While this discussion is quite general in nature, it is important to note that Sgr A East, a mixed-morphology SNR located within several parsecs of the Galactic center, is a viable candidate for the site of hadronic acceleration (see $\S 7.2$ for a more complete discussion). Specifically, the observation of $\mathrm{OH}$ maser emission at the boundary of this structure provides strong evidence for the presence of shocks (Yusef-Zadeh et al. 1996, 1999). In addition, Fatuzzo \& Melia (2003) have found that a power-law distribution of shock-accelerated relativistic protons injected into the high-density, strongly magnetized Sgr A East enviroment leads to a pion-decay process (described above) that can account for both the EGRET source 3EG J1746-2851 and the unique radio characteristics of Sgr A East. This scenario may also account for the $e^{+}-e^{-}$annihilation radiation observed from the galactic bulge by the Oriented Scintillation Spectrometer Experiment (OSSE) aboard the Compton Gamma Ray Observatory (Fatuzzo, Melia and Rafelski 2001).

Finally, further evidence for the occurence of hadronic acceleration at the Galactic center is presented by the detection of this region at $\sim \mathrm{TeV}$ energies by a number of air Cerenkov telescopes: see $§ 5.4$ for more on this point.

\section{Relating a Pion-Decay $\gamma$-ray flux to a Neutron Flux}

The processes via which an impinging, EHE charged beam can lead to the production of astrophysical neutrons can be summarized as (with the 'beam' particle indicated first in each pair, the target second):
1. $\mathrm{pp}$
2. Ap, $\mathrm{A} \neq \mathrm{p}$
3. $\mathrm{p} \gamma$
4. $\mathrm{A} \gamma, \quad \mathrm{A} \neq \mathrm{p}$. 
In more detail, these processes are:

1. Leading neutron production from collisions of accelerated protons with ambient, target protons.

2. Neutron production via dissociation of accelerated ions through collisions with ambient, target protons.

3. Photo-production of leading neutrons (i.e., charge-exchange production of neutrons in collisions of accelerated protons with ambient, target photons).

4. Photo-dissociation (fragmentation) of accelerated ions.

Of the existing work concerned with the GC anisotropy and its explanation in terms of neutron production at various possible GC sites, the AGASA group's anisotropy discovery paper (Hayashida et al. 1999a) focused on the idea that the disintegration of accelerated heavy ions by interactions with ambient matter or photons was the ultimate source of the (putative) EHE neutrons. This follows the lines of the broad scenario investigated by Sikora (1989) for active galactic nuclei in which the very same strong magnetic fields that serve to accelerate charged particles to high energies also serve to confine the same to some central accelerating region (whereas neutrons escape). Also of relevance here is the work of Tkaczyk (1994).

Alternatively to the heavy-ion disintegration idea, Medina-Tanco and Watson (2001) have proposed that a more likely method of EHE neutron production is via interactions between accelerated protons and ambient protons or IR $\gamma$ 's. They found that the environment of Sgr $A^{*}$ - the supermassive black hole at the GC - is sufficiently dense that the particle interaction rate required to produce the desired neutron flux is achievable. We shall have more to say about this scenario below.

Takahashi and Nagataki (2001) also considered neutron production and determined that it is pp interactions which most effectively produce the required neutrons. For reasons we shall explain below, we agree with this conclusion (though our calculations differ importantly in specifics). Takahashi and Nagataki (2001) also researched the detectability of neutrinos concomittant with neutron production. More recently, Anchordoqui et al. (2003) have studied the detectability of neutrinos produced in the decay-in-flight of the (putative) GC neutron beam and Biermann et al (2004) have considered a model in which the observed anisotropy is explained as due to the last GRB to go off in the Galaxy. 


\subsection{Detailed Calculation of Neutron Flux from pp Collisions}

Protons accelerated to relativistic energies at the GC source can undergo a series of interactions including $p N \rightarrow p N m_{\text {meson }} m_{N \bar{N}}$, where $N$ is either a $p$ or a neutron $n, m_{\text {meson }}$ denotes the energy-dependent multiplicity of mesons (mostly pions), and $m_{N \bar{N}}$ is the multiplicity of nucleon/anti-nucleon pairs (both increasing functions of energy). Since $m_{N \bar{N}} / m_{\text {meson }}<10^{-3}$ at low energy and even smaller at higher energies (Cline 1988), following Markoff et al. (1997) we here ignore the anti-nucleon production events. The charge exchange interaction $(p \rightarrow n)$ occurs around $40 \%$ of the time at accelerator energies and this fraction is predicted to be only very weakly energy-dependent (see the appendix). We shall take it, then, that the leading neutron multiplicity, $m_{n}$, is given by a fixed proportion of 0.4 (i.e., $40 \%$ of all pp interactions involve charge exchange, independent of incoming proton energy).

Other possible interactions of accelerated $p$ 's - all potentially important for cooling are $p \gamma \rightarrow p \pi^{0} \gamma, p \gamma \rightarrow n \pi^{+} \gamma, p \gamma \rightarrow e^{+} e^{-} p$ and $p e \rightarrow e N m_{\text {meson }}$ (Markoff et al. 1997).

\subsubsection{The production of $\pi^{0}$ decay photons}

The (differential) $\pi^{0}$ emissivity resulting from an isotropic distribution of shock accelerated protons $d n_{p}\left(E_{p}\right) / d E_{p}$ (where $\left[d n_{p}\left(E_{p}\right) / d E_{p}\right]$ is in units of $\mathrm{cm}^{-3} \mathrm{eV}^{-1}$ ) interacting with cold (fixed target) ambient hydrogen of density $n_{H}$ is given by the expression

$$
Q_{\pi^{0}}^{p p}\left(E_{\pi^{0}}\right)=c n_{H} \int_{E_{p}^{t h}\left(E_{\pi^{0}}\right)} d E_{p} \frac{d n_{p}\left(E_{p}\right)}{d E_{p}} \frac{d \sigma\left(E_{\pi^{0}}, E_{p}\right)}{d E_{\pi^{0}}}
$$

where $E_{p}^{t h}\left(E_{\pi^{0}}\right)$ is the minimum proton energy required to produce a pion with total energy $E_{\pi^{0}}$ (determined through kinematical considerations) and $\left[Q_{\pi^{0}}^{p p}\right]=$ pions s${ }^{-1} \mathrm{~cm}^{-3} \mathrm{eV}^{-1}$. The resulting $\gamma$-ray emissivity is then given by the expression

$$
Q_{\gamma}\left(E_{\gamma}\right)=2 \int_{E_{\pi^{0}}^{\min }\left(E_{\gamma}\right)} d E_{\pi^{0}} \frac{Q_{\pi^{0}}^{p p}\left(E_{\pi^{0}}\right)}{\left(E_{\pi^{0}}^{2}-m_{\pi^{0}}^{2}\right)^{1 / 2}}
$$

where $E_{\pi^{0}}^{\min }\left(E_{\gamma}\right)=E_{\gamma}+m_{\pi^{0}}^{2} /\left(4 E_{\gamma}\right)$.

At proton energies, $E_{p}$, greater than $\sim 5 \mathrm{GeV}$ - above the $\Delta$ resonance-affected regionthe differential cross-section is approximated by the scaling form of Blasi \& Melia (2003; see also Blasi \& Colafrancesco 1999):

$$
\frac{d \sigma\left(E_{p}, E_{\pi^{0}}\right)}{d E_{\pi^{0}}}=\frac{\sigma_{0}}{E_{\pi^{0}}} f_{\pi^{0}}\left(x_{0}\right)
$$


where $x_{0} \equiv E_{\pi^{0}} / E_{p}, \sigma_{0}=32$ mbarn, and (Hillas 1980)

$$
f_{\pi^{0}}\left(x_{0}\right)=0.67\left(1-x_{0}\right)^{3.5}+0.5 e^{-18 x_{0}}
$$

This scaling form properly takes into account the high pion multiplicities which occur at high energies.

Given the above and a parent proton distribution governed by a power law, $d n_{p}\left(E_{p}\right) / d E_{p}$ with spectral index $\gamma$,

$$
\frac{d n_{p}\left(E_{p}\right)}{d E_{p}} \propto E_{p}^{-\gamma}
$$

we can write the neutral pion emissivity due to pp as

$$
Q_{\pi^{0}}^{p p}\left(E_{\pi^{0}}\right)=\frac{d n_{p}\left(E_{\pi^{0}}\right)}{d E_{p}} \sigma_{0} n_{H} c \Lambda^{0}(\gamma)
$$

and, consequently, the photon emissivity due to the decay of these $\pi^{0}$ 's as

$$
Q_{\gamma}\left(E_{\gamma}\right) \simeq c n_{H} \int_{E_{\gamma}}^{\infty} d E_{\pi^{0}} \int_{E_{\pi^{0}}}^{\infty} d E_{p} \frac{d n_{p}\left(E_{p}\right)}{d E_{p}} \frac{d \sigma\left(E_{p}, E_{\pi^{0}}\right)}{d E_{\pi^{0}}} \frac{2}{E_{\pi^{0}}} \simeq \frac{2}{\gamma} \frac{d n_{p}\left(E_{\gamma}\right)}{d E_{p}} \sigma_{0} n_{H} c \Lambda^{0}(\gamma)
$$

where in both equations immediately above we employ

$$
\Lambda^{0}(\gamma) \equiv \int_{0}^{1} d x_{0} x_{0}^{\gamma-2} f_{\pi^{0}}=2\left\{\Gamma(\gamma-1)\left[18^{1-\gamma}+\frac{15.5865}{\Gamma(3.5+\gamma)}\right]-E(2-\gamma ; 18)\right\}
$$

in which $\Gamma(x)$ is the Euler Gamma function and $E(n ; z)$ is the exponential integral function which satisfies $E(n ; z) \equiv \int_{1}^{\infty} \exp (-z t) / t^{n} \mathrm{~d} t$.

\subsubsection{The Production of Neutrons in the Scaling Regime}

Similarly to the above, the emissivity of neutrons from an isotropic distribution of shock accelerated protons $d n_{p}\left(E_{p}\right) / d E_{p}$ interacting with cold (fixed target) ambient hydrogen of density $n_{H}$ is given by the expression

$$
Q_{n}^{p p}\left(E_{n}\right)=c n_{H} \int_{E_{p}^{t h}\left(E_{n}\right)} d E_{p} \frac{d n_{p}\left(E_{p}\right)}{d E_{p}} \frac{d \sigma\left(E_{n}, E_{p}\right)}{d E_{n}}
$$

To proceed further we would like to take, in analogy to the above,

$$
\frac{d \sigma\left(E_{p}, E_{n}\right)}{d E_{n}}=\frac{\sigma_{0}}{E_{n}} f_{n}\left(x_{n}\right)
$$


where $x_{n} \equiv E_{n} / E_{p}$. We must now determine an expression for $f_{n}\left(x_{n}\right)$. In this regard, we employ the formalism set out in Appendix A of Drury et al. (1994). Following Gaisser (1990), this reference sets out the calculation of the 'spectrum-weighted moment' (SWM), denoted by $\left\langle m x_{S}\right\rangle_{S}^{\gamma}$, for the emission spectrum of various particle species (labeled by $S$ ) from collisions of protons from a power-law distribution equation (7) and where $x_{S} \equiv E_{S} / E_{p}$. In this formalism, the emissivity of species $S$ can be written

$$
Q_{S}\left(E_{S}\right)=\frac{d n_{p}\left(E_{S}\right)}{d E_{p}} \sigma_{p p} n_{H} c\langle m x\rangle_{S}^{\gamma}
$$

so that we see that $\Lambda^{0}(\gamma)$ defined in equation (10) above is nothing but the SWM for neutral pions produced in pp collisions.

Drury et al. (1994) provide their own calculation of the SWM for neutral pions, decay gammas, and neutrons $\left(\langle m x\rangle_{\pi^{0}}^{\gamma},\langle m x\rangle_{\gamma}^{\gamma}\right.$, and $\left.\langle m x\rangle_{n}^{\gamma}\right)$. We set out their results and ours (calculated with the $f_{\pi^{0}}$ given above) for comparison for pions and pion decay gammas in Table 1.

To arrive at a SWM for neutron production calculations, Drury et al. (1994) employ the dimensionless, inclusive cross-section for neutron production given by Jones (1990), viz

$$
g_{n}\left(x_{n}\right)=n_{n}\left(\alpha_{n}+1\right)\left(1-x_{n}\right)^{\alpha_{n}} .
$$

Jones (1990) gives, on the basis of his analysis of $300 \mathrm{GeV}$ proton collider data, $\alpha_{n}=2$, and an average neutron multiplicity, $n_{n}$, of 0.25 . We adopt his results excepting the neutron multiplicity which we revise to be 0.4: see the appendix for more detail on this issue. We find, then, that

$$
g_{n}\left(x_{n}\right) \rightarrow 1.2\left(1-x_{n}\right)^{2} .
$$

The translation of this distribution into our formalism is simply

$$
f_{n}\left(x_{n}\right) \equiv x_{n} g_{n}\left(x_{n}\right)=1.2 x_{n}\left(1-x_{n}\right)^{2} \text {. }
$$

We find, then,

$$
Q_{n}^{p p}\left(E_{n}\right) \simeq c n_{H} \sigma_{0} \frac{d n_{p}\left(E_{n}\right)}{d E_{p}} \Lambda^{n}(\gamma)
$$

where we have defined

$$
\Lambda^{n}(\gamma) \equiv \int_{0}^{1} d x_{n} x_{n}^{\gamma-2} f_{n}\left(x_{n}\right)=2.4 \frac{1}{\gamma(\gamma+1)(\gamma+2)}
$$




\begin{tabular}{|c|cccc|}
\hline$\gamma$ & 2.0 & 2.2 & 2.4 & 2.6 \\
\hline$\Lambda^{0}(\gamma)$ & 0.177 & 0.113 & 0.076 & 0.053 \\
$\langle m x\rangle_{\pi^{0}}^{\gamma}$ & 0.17 & 0.092 & 0.066 & 0.048 \\
\hline$\Lambda^{n}(\gamma)$ & 0.177 & 0.103 & 0.063 & 0.041 \\
$\langle m x\rangle_{n}^{\gamma}$ & 0.19 & 0.094 & 0.051 & 0.030 \\
\hline
\end{tabular}

Table 1: Values of $\Lambda(\gamma)$ for both neutral pions and neutrons from pp collisions compared to the spectrum-weighted distributions for same calculated in Drury et al. (1994).

\subsubsection{Relating Photon and Neutron Emissivity and Fluxes in the Scaling Regime}

Now, from Eqs.(9) and (17) we have that

$$
Q_{n}^{p p}\left(E_{n}\right)=0.8 \gamma \frac{\Lambda^{n}(\gamma)}{\Lambda^{0}(\gamma)} Q_{\gamma}\left(E_{\gamma}^{0}\right)\left(\frac{E_{n}}{E_{\gamma}^{0}}\right)^{-\gamma}
$$

where we have also used the fact that both daughter $\gamma$ and neutron spectra will be governed by the same power law as the parent protons. We have, then, related the neutron emissivity at some energy $E_{n}$ to the photon emissivity at a normalization energy $E_{\gamma}^{0}$ (assuming scaling holds). We present $\left(0.8 \gamma \Lambda^{n}[\gamma]\right) / \Lambda^{0}[\gamma]$ for various values of the spectral index $\gamma$ in Table 2 . The growth of this ratio with energy can be related to the fact that, because the average

energy of a neutron produced in a pp interaction (at lab energy $E_{p}$ ) will be higher than the energy of a pion-decay $\gamma$ produced (indirectly) by a pp interaction at the same energy $\left(E_{p}\right)$, the neutron flux at $E_{n}$ is 'directly' tied to the photon flux at a somewhat lower energy. This means that as one steepens a spectrum - thereby increasing the number of photons in the population below some fixed pivot point (at which the normalization is effected) - one also tends to increase the population of neutrons at $E_{n}$.

Note that in employing the relation set out in equation (19), careful attention should be paid to the following points:

1. The neutron emissivity is related not to the total photon emissivity but, rather, related to that part of the photon signal due to pion decay. Determining an expectation, then, for a neutron emissivity or flux requires that one be able to confidently pin down what proportion of the $\gamma$-ray signal at some normalization energy is due to pion decay.

2. In our derivation it was assumed that $f_{\pi^{0}}(x)$ is given by its scaling form. This means that photon emissivity (or flux) data must be taken from observations made at sufficiently high energy that we are guaranteed to be in the scaling regime, viz above $\sim 5$ $\mathrm{GeV}$. 
3. Likewise, it is assumed that the neutrons scale as indicated by equation (16). This relation can be expected to go wrong at sufficiently high energies (see below for more on this point). Note that we are concerned with pp interactions at a lab energy of $\sim 5 \times 10^{18} \mathrm{eV}$ entailing a cms energy range of $\sqrt{s} \sim 70 \mathrm{TeV}$.

4. The parent proton power law will cut off at sufficiently high energy. In making predictions for neutron flux one must check that one is not above this cut-off energy.

\begin{tabular}{|c|ccccc|}
\hline$\gamma$ & 2.0 & 2.1 & 2.2 & 2.4 & 2.6 \\
\hline$\left(\gamma \Lambda^{n}[\gamma]\right) /\left(2 \Lambda^{0}[\gamma]\right)$ & 0.566 & 0.674 & 0.790 & 1.072 & 1.360 \\
\hline
\end{tabular}

Table 2: The ratio between photon and neutron emissivities - at the same energy - from pp collisions in the scaling regime.

\subsection{The Production of Neutrons at EHE: Accounting for Cross-Section Scaling Violation}

The above technology allows us to relate the neutron emissivity to the photon emissivity of the same astrophysical object provided that scaling holds. That this caveat obtains can be seen directly from the fact that the integral of equation (12) over $E_{n}$ - which should define the inclusive cross-section for neutron production - is, in fact, independent of center-of-mass energy. This approximation holds good, by definition, over the scaling regime from, say, 10 to $1000 \mathrm{GeV}$ for the incident proton energy in the lab frame, but at the lab energies of over $10^{9} \mathrm{GeV}$ that we are concerned with for EHE neutron production, it is no longer accurate (see Hagiwara et al. 2002, fig. 39.12). To the level of accuracy required for the current application, however, it is not too difficult to account for the cross-section growth. To render the logic here most perspicuous, the process can be described as a two-step one: (i) relate, via equation (19), the photon and neutron emissivities at an energy scale small enough that scaling holds and the cross-section can be taken to be constant with respect to center-of-mass energy (ii) relate the high energy neutron emissivity to the low energy neutron emissivity (inside the scaling regime) via the assumed power-law distribution and the ratios of the total pp cross-sections at these two energy regimes, $\sigma_{p p}\left(E_{n}\right) / \sigma_{p p}\left(E_{\gamma}\right)$.

\subsection{Neutron and $\gamma$-Ray Fluxes Related Given Neutron Decay}

Of course, we can equally well relate the observed pion decay $\gamma$-ray flux at the Earth to the expected neutron flux (neglecting neutron decay - and other effects that might treat 
photons and neutrons differently in propagation - for the moment). Indeed, one may quickly determine that, given a power-law parent proton distribution, and also accounting for the growth in the total cross-section discussed above,

$$
F_{n}^{n . d .}\left(E_{n}\right) \simeq 0.8 \gamma \frac{\Lambda^{n}(\gamma)}{\Lambda^{0}(\gamma)} F_{\gamma}\left(E_{\gamma}^{0}\right)\left(\frac{E_{n}}{E_{\gamma}^{0}}\right)^{1-\gamma} \frac{\sigma_{p p}\left(\sim 10^{18} \mathrm{eV}\right)}{\sigma_{p p}\left(\sim 10^{12} \mathrm{eV}\right)}
$$

where $F_{n}^{n . d .}\left(E_{n}\right)$ denotes the total flux of neutrons above $E_{n}$ that would be detected at Earth if the neutrons did not decay (n.d. denotes 'no decay') and $F_{\gamma}\left(E_{\gamma}^{0}\right)$ denotes the total flux of $\pi$ decay photons detected above the normalization energy $E_{\gamma}^{0}$. Note that the above relationdirectly relating a neutron flux at Earth to a photon flux at Earth-obviates the need for an accurate estimate of the distance to the source. Also note from fig. 39.12 of Hagiwara et al. (2002) that the ratio of the pp total cross-sections pertinent to the $\gamma$-ray and neutron production regimes is

$$
\frac{\sigma_{p p}\left(\sim 10^{18} \mathrm{eV}\right)}{\sigma_{p p}\left(\sim 10^{12} \mathrm{eV}\right)} \simeq \frac{150 \mathrm{mb}}{40 \mathrm{mb}}=3.75
$$

We are now in a position to provide a preliminary estimate of the neutron flux above, say, $10^{17.9} \mathrm{eV}$ based on the EGRET data on the GC source. From the data provided in Mayer-Hasselwander et al. (1998), the differential flux of photons at $\sim 6.3_{-2.3}^{+3.7} \times 10^{9} \mathrm{eV}$ is $6 \times 10^{-18} \mathrm{~cm}^{-2} \mathrm{~s}^{-1} \mathrm{eV}^{-1}$. With a power-law spectrum of spectral index $\gamma$ this translates to a gamma ray flux

$$
F_{\gamma}\left(6.3 \times 10^{9} \mathrm{eV}\right)=\frac{\left(3.8_{-1.8}^{+3.2}\right)}{\gamma-1} \times 10^{-8} \mathrm{~cm}^{-2} \mathrm{~s}^{-1} .
$$

Substituting this photon flux into equation (20) we find the values for neutron flux tabulated against $\gamma$ in the first row of Table 3 .

As stressed, however, these preliminary flux estimates do not take into account propagation effects. In this regard, we can quickly dismiss any potential effect from attenuation of the neutrons due to collisions with ambient particles in propagation: here the greatest potential effect would be due to collisions of the neutrons with ambient protons (see $§ 6.3 .1$ below). Now, based on extinction at IR and optical wavelengths, the column density to the GC is at most around $5 \times 10^{22} \mathrm{~cm}^{-2}$, implying a 'grammage' of matter that must be traversed by the GC neutrons in reaching the Earth of around $0.1 \mathrm{~g} \mathrm{~cm}^{-2}$ or less. Neutron energy losses due to interaction with ambient matter in propagation only become significant for grammages in excess of $80 \mathrm{~g} \mathrm{~cm}^{-2}$ (Tkaczyk 1994), however, and are, therefore, entirely negligible in this instance (one can also establish that $\mathrm{TeV}$ photons from the $\mathrm{GC}$ are not significantly attenuated by pair production in propagation: see $\S 6.1$ ).

In contrast, neutron decay must certainly be accounted for. We can incorporate this 
effect by writing the flux as

$$
F_{n}\left(E_{n}\right) \simeq \int_{E_{n}}^{\infty} \frac{\mathrm{d} F_{n}^{\text {n.d. }}\left(E_{n}\right)}{\mathrm{d} E_{n}} \exp \left(-\frac{d_{G C}}{d_{n}\left[E_{n}\right]}\right) \mathrm{d} E_{n},
$$

where $d_{G C}$ is the distance to the Galactic Center, $d_{n}(E)$ is the neutron mean free path defined in equation (1) and the neutron differential flux without decay is given by (cf. equation 19)

$$
\frac{\mathrm{d} F_{n}^{\text {n.d. }}\left(E_{n}\right)}{\mathrm{d} E_{n}} \simeq 3.0 \gamma \frac{\Lambda^{n}(\gamma)}{\Lambda^{0}(\gamma)} \frac{\mathrm{d} F_{\gamma}\left(E_{\gamma}^{0}\right)}{\mathrm{d} E_{\gamma}^{0}}\left(\frac{E_{n}}{E_{\gamma}^{0}}\right)^{-\gamma},
$$

in which, as above, $E_{\gamma}^{0}$ is some normalizing energy at which the $\pi^{0}$-decay photons are observed and the numerical factors from equations (20) and (21) combine to give $3.75 \times 0.8=3.0$. Note that now the distance to the source enters into the calculation. We present the result of calculating the neutron flux with decay in the second line of Table 3. As one can see from

\begin{tabular}{|c|cccc|}
\hline$\gamma$ & 2.1 & 2.2 & 2.3 & 2.4 \\
\hline (i) & $\left(1.1_{-0.7}^{+1.7}\right) \times 10^{-16}$ & $\left(1.7_{-1.1}^{+3.1}\right) \times 10^{-17}$ & $\left(3.0_{-1.9}^{+5.6}\right) \times 10^{-18}$ & $\left(4.9_{-3.2}^{+9.9}\right) \times 10^{-19}$ \\
(ii) & $\left(6.1_{-3.7}^{+9.9}\right) \times 10^{-17}$ & $\left(9.8_{-6.1}^{+17.3}\right) \times 10^{-18}$ & $\left(1.6_{-1.0}^{+3.0}\right) \times 10^{-18}$ & $\left(2.6_{-1.7}^{+5.2}\right) \times 10^{-19}$ \\
\hline
\end{tabular}

Table 3: Values for the flux of neutrons above $10^{17.9} \mathrm{eV}$ due to the EGRET GC source for the cases where neutron decay-in-flight (i) is not and (ii) is accounted for. The units are neutrons $\mathrm{cm}^{-2} \mathrm{~s}^{-1}$. These numbers should be compared to the flux calculated 'indirectly' from the AGASA data by (4), viz. $2 \times 10^{-17} \mathrm{~cm}^{-2} \mathrm{~s}^{-1}$ between $10^{17.9} \mathrm{eV}$ and $10^{18.5} \mathrm{eV}$, and 'directly' from the SUGAR data for the observed point-like excess, viz $\left(9 \pm 3 \times 10^{-18}\right) \mathrm{cm}^{-2}$ $\mathrm{s}^{-1}$ for the same energy range (Bellido et al. 2001).

Table 3, the putative neutron source seen (indirectly) by AGASA and (directly) by SUGAR is easily accounted for by the GC EGRET source with a spectral index of $\gamma=2.2 \rightarrow 2.3$ (the best fit is at 2.23: see §6.1). Not only is this range full compatible with the expectation from theoretical calculations of shock acceleration spectra, it is also completely consistent with the value of 2.2 recently determined by Fatuzzo and Melia (2003) as their best fit for pion-decay $\gamma$ 's to the EGRET source 3EG J1746-2851 (see more on this point below) and, further, is also consistent with the spectral index of 2.2 determined by Bossa et. al (2003) in their fit to the EHE AGASA data. Note that other possible neutron production channels (besides pp collisions) are discussed in $§ 6.3$.

\subsection{Detection of the Galactic Center at TeV Energies}

A Galactic center source has been detected by three air Čerenkov telescopes (ACTs) at $\mathcal{O}[\mathrm{TeV}]$ energies: Whipple (Kosack et al. 2004), CANGAROO (Tsuchiya et al. 2004), and, 
most recently and significantly, HESS (Aharonian et al. 2004). In addition, the Hegra ACT instrument has put a (weak) upper limit on GC emission at 4.5 GeV (Aharonian et al. 2002) and the Milagro water Čerenkov extensive air-shower array has released a preliminary finding of a detection at similar energies from the 'inner Galaxy' (defined as $l \in\left\{20^{\circ}, 100^{\circ}\right\}$ and $|b|<5^{\circ}$ : Fleysher (2002)). Here we address only the Whipple, CANGAROO, and HESS results in any detail, though note that all the observations mentioned above lend crucial support to the notion that acceleration of particles to very high energies is taking place at the GC.

Even restricting ourselves to consideration of results from these three instruments, we find the situation somewhat confused regarding the GC. In fact, it was clear, even before the arrival of the recent, remarkable data from the HESS instrument, that the Whipple and CANGAROO GC observations were in conflict: Whipple has detected (in data collected over a total of 26 hours from 1995 to 2003), at (conservatively) the $3.7 \sigma$ level, a flux of photons from the GC direction of $1.6 \pm 0.5$ stat $\pm 0.3 \mathrm{sys} \times 10^{-12} \mathrm{~cm}^{-2} \mathrm{~s}^{-1}$ above $2.8 \mathrm{TeV}$ (Kosack et al. 2004) which is $40 \%$ of the Crab flux above this same energy. (In regards to the errors on the measuremnt, note that there is a $20 \%$ uncertainty, too, in the callibration of the $2.8 \mathrm{TeV}$ energy threshold). This is to be contrasted with GC data which were collected in 2001 and 2002 by the CANGAROO-II ACT. From these data the CANGAROO collaboration has been able to generate a spectrum for the GC source in six energy bins. From this spectrum can be extracted (Hooper et al. 2004) a flux of around $2 \times 10^{-10} \mathrm{~cm}^{-2} \mathrm{~s}^{-1}$ above $250 \mathrm{GeV}$ : this is at the level of $10 \%$ of the Crab (Tsuchiya et al. 2004). The CANGAROO Collaboration also determine an extremely steep spectrum for their GC source with a fitted spectral index of $4.6 \pm 0.5$.

The fluxes of the Whipple and CANGAROO sources (relative to the Crab) are, then, quite different and a natural reading of the situation is that the instruments are in conflict (see Hooper et al. (2004) for further discussion on this point). Note that any variability of the GC source at these energies is now constrained to be small (Kosack et al. 2004) so that, though the two instruments in question collected their GC data over periods largely noncoincident with each other, there is little leeway for explaining the difference between the two instruments by positing that they happened to observe the source at different activity levels. Also note that the fields-of-view of the two instruments are similar and that their respective GC sources are at similar positions and of similar extent ${ }^{5}$.

Adding very significantly to our knowledge of the $\mathrm{GC}$ at $\sim \mathrm{TeV}$ energies, the High Energy Stereoscopic System (HESS) Collaboration (Hinton 2004), which employs four imaging

\footnotetext{
${ }^{5}$ Steve Fegan, private communication.
} 
atmospheric Cerenkov telescopes, has recently released TeV-range, GC data unprecedented in its detail. This group has detected a signal in observations conducted over two epochs (June/July 2003 and July/August 2003) with a $6.1 \sigma$ excess evident in the former and a 9.2 $\sigma$ excess in the latter (Aharonian et al. 2004). The data from the larger, July/August 2003 data set (which we shall use in our analysis) can be fitted by a power law with, from the collaboration's own determination (Aharonian et al. 2004), a spectral index $2.21 \pm 0.09$ and normalization $(2.50 \pm 0.21) \times 10^{-8} \mathrm{~m}^{-2} \mathrm{~s}^{-1} \mathrm{TeV}^{-1}$ with a total flux above the instrument's $165 \mathrm{GeV}$ threshold of $(1.82 \pm 0.22) \times 10^{-7} \mathrm{~m}^{-2} \mathrm{~s}^{-1}$ (there is also a $15-20 \%$ error from energy resolution uncertainty). Data from the June/July 2003 run are consistent within errors with the July/August 2003 data.

The HESS flux determination is equivalent to $5 \%$ of that from the Crab above the 165 $\mathrm{GeV}$ threshold. It is in conflict with the results from Whipple (see figures 1 and 2), the latter's flux determination being a factor of three above that implied by the HESS spectrum (Aharonian et al. 2004). It is also in striking conflict with the CANGAROO data: the HESS spectral index determination is clearly at variance with the very steep spectrum found by CANGAROO (see figure 4 of Aharonian (2004) for a clear illustration of this fact). Again, one might interpret these discrepancies as evidence for significant source variation between the the instruments' different observing periods (i.e., over timescales of $\sim$ year) but the multimonth HESS data no more indicate source variability within the observing period than the previous observations (Aharonian et al. 2004).

Intriguingly, the HESS data are also difficult to reconcile with the EGRET GC data (again, see figures 1 and 2 and also see the inset of figure 4 of Aharonian(2004)) - if one assumes a single source. We shall have more to say about this issue below. For the moment, however, we must clearly decide upon which of the TeV data sets we should base our analysis. Certainly one telling point against the CANGAROO data, as noted above, is the unusually steep, GC source spectrum. Although we do not pretend to any in-depth knowledge of the workings of the CANGAROO analysis, we do note that observations of instrinsically bright regions like the GC are, generically, affected by the problem that at lower energies putative events can be 'bumped over' a detector's threshold by noise, whereas at higher energies such a mechanism would not be expected to be working ${ }^{6}$. A source spectrum, then, might

\footnotetext{
${ }^{6}$ One way around this potential problem is to employ a 'padding' procedure in which artificial noise is fed into the data to account for the systematic brightness differences between on-source and background observations. This is at the cost of reducing the signal-to-noise ratio and, therefore, raising the threshold for observations but without such a procedure the artifical steepening of a power law is difficult to avoid. Now, the VERITAS Collaboration, in its analysis of the Whipple data from sources located in bright regions employs exactly the padding procedure described immediately above (Steve Fegan, private communication).
} 
be made to seem steeper than in actuality by this preferential recording of lower energy events. Furthermore, though the HESS and Whipple data are somewhat at variance, they are certainly less in conflict than either result is with CANGAROO (again, see figure 4 of Aharonian (2004)), so they support each other in a qualified sense. For these reason we focus on the HESS and Whipple results and, then noting both the greater detail and statistical weight in the former, we finally are drawn to the conclusion that we should leave only the HESS data in our analysis.

\section{Fitting to All Data}

We will now attempt to derive a best fit to the following data: the EGRET $\gamma$-ray differential flux at $\sim \mathrm{GeV}$ energies, the HESS $\sim \mathrm{TeV} \gamma$-ray flux and the EHE $\left(\sim 10^{18} \mathrm{eV}\right)$ cosmic ray anisotropy data (assumed due to neutrons). Such a fitting procedure makes sense in principle because, as shown above, the neutron and photon fluxes are governed by power laws with the same spectral index (as both these species arise from the interactions of the same parent spectrum of accelerated protons) and at any energy we can relate the flux or differential flux of neutrons and photons.

Our procedure is to define a $\chi^{2}$ function which depends on the differences between fitted and observed fluxes or diffential fluxes, weighted by the experimental error in each flux measurement and also allowing for the systematic uncertainty in the energy calibration of the various instruments. The free parameters in our analysis are the spectral index, $\gamma$, and the normalization of the $\gamma$-ray differential flux. We describe our procedure at greater length in an appendix.

Employing this procedure, one quickly learns that the hypothesis that the totality of $\gamma$-ray and cosmic ray data can be explained as arising from the interactions of a single, parent population of shock-accelerated protons is not supported by the data: the fit procedure produces a $\chi^{2}$ of 78.55 with a reduced $\chi^{2}$ of 6.54 for the $(14-2)$ degrees of freedom (dof) which is a very bad fit. There are a number of caveats to this bald assertion, however, which we shall explore in somewhat greater detail below. These are, firstly, that it assumes that the detected radiation - in its various energy regimes - is all equally unaffected in its propagation. A process which, say, attenuated $\gamma$-rays at $\mathrm{TeV}$ energies but not at $\mathrm{GeV}$ energies might be operating but this is not accounted for in the fitting procedure. Secondly, the fits assumes that there is, effectively, only a single source. The GC is a highly energetic and dense region;

But, to the best of our knowledge, the CANGAROO collaboration does not use this procedure. For this reason we believe that the slope of the CANGAROO data on the GC source are questionable. 
there could, in reality, then, be a number of effective sources or acceleration/interaction regions (characterized by different magnetic field strengths, different shock compression ratios, different shock/magnetic field geometries, different ambient particle densities, etc) there. Thirdly, the fit procedure assumes that the detected particles/radiation all originate in the same pp collision process. If, say, another process were operating at high energies to supply some significant fraction of the observed neutrons, then accounting for this process might allow for a better fit to the totality of data (that said, the EGRET and HESS data are still difficult to reconcile). We now explore these various points in greater detail.

\subsection{A Single Source: Attenuation of TeV Photons?}

As should be expected from $\S 5.3$, if one performs a fit to only the EGRET+EHECR data ('EHECR' denotes extremely high energy cosmic ray': it is implicit in our analysis, of course, that these data are explained as arising from neutron primaries), neglecting the HESS data, one finds a very good fit: the total $\chi^{2}$ is 0.33 for $(4-2)$ degrees of freedom for a very plausible spectral index of 2.23. We remind the reader that this value is perfectly consistent with that determined by Fatuzzo and Melia (2003) for the EGRET source 3EG J1746-2851 and by Bossa, Mollerach, and Roulet (2003) for the EHE CR source seen by AGASA (both these reference quote a figure of 2.2) and is, moreover, perfectly consonant with the expectation from theory for acceleration at strong shocks. Further, the fitting points are around eight orders of magnitude apart so it is, indeed, remarkable that the fit obtained between such widely-separated data points should require a spectral index so close to the expectation from shock acceleration theory and previous observations in more limited energy regimes (see the upper line in figure 1).

The one problem with this scenario is the significant (by a factor of $\sim 20$ ) overprediction of $\mathrm{TeV} \gamma$-rays (again, see figure 1). We note that there is no question but that our fit to the EGRET+EHECR data does predict a TeV source which is inside the field of view of HESS's GC observations (and Whipple's for that matter) and should have been seen by this instrument. The simplest possibility to resolve the discrepancy, which we respectfully submit, is that the normalization of HESS GC data is simply off by a large number. In support of this, note that the other remarkable aspect of figure 1 is that the lower line which is a fit to the July/August 2003 HESS data alone - so closely parallels the upper: it has a best-fit spectral index of $2.22^{7}$. It does seem rather unlikely, however, that a normalization

\footnotetext{
${ }^{7}$ This spectral index has been independently re-derived by us: the HESS group find a spectral index of $2.21 \pm 0.09$ with a normalization of $2.510^{-8} \mathrm{~m}^{-2} \mathrm{~s}^{-1} \mathrm{TeV}^{-1}$ at $1 \mathrm{TeV}$. We perfectly agree with this
} 
error could really be as large as we require it and (given also that even the larger Whipple flux determination is still deficient with respect to our expectation from the single source model) we are compelled to seek astrophysical explanations of the data considering them to be fundamentally sound.

One possibility, presaged above, is that some process is acting to attenuate or downgrade the energies of the $\sim \mathrm{TeV}$ photons after they have been generated, i.e., in their propagation from interaction point to us. In this regard, probably the most attractive mechanism is pair-production on the optical-NIR background near the source. Certainly something like this process is known to operate in the "self-absorption" of $\mathrm{TeV} \gamma$-rays from some X-ray binary systems by the thermal photons emitted by those systems' own accretion disks (see Moskalenko (1995) for a review). Pair production has an effective threshold which means that it does not significantly attenuate $\gamma$-rays with energies below a threshold, $E_{\gamma}^{\text {thresh(pair) }}$, given roughly by

$$
E_{\gamma}^{\text {thresh(pair) }} \sim 1\left(\frac{E_{\text {bckgnd }}}{0.5 \mathrm{eV}}\right)^{-1} \mathrm{TeV},
$$

where $E_{\text {bckgnd }}$ is the typical energy of the (relevant) background photon population. With NIR-optical background light, then, the GC GeV signal would remain unattenuated as desired. From equation (25) we require a background of $\sim 1.5 \mathrm{eV}$ (i.e., NIR-optical) photons to attenuate the HESS signal right down to the lowest datum at around $300 \mathrm{GeV}$. Further considerations are the following:

1. Attenuation of the signal by $\sim 1 / 20$ of the expectation (given the fit to the other data) implies an optical depth of $\ln (20) \sim 3$

2. The peak cross-section for pair production is roughly $10^{-25} \mathrm{~cm}^{2}$, so we require a column density of photons of around $3 / 10^{-25} \mathrm{~cm}^{2}=3 \times 10^{25} \mathrm{~cm}^{2}$ to attenuate the photons. Over the entire $8.5 \mathrm{kpc}$ to the GC this would require an average optical-NIR photon number density of $\sim 1000 \mathrm{~cm}^{3}$. This is orders of magnitude larger than what is found in the Galactic plane, so we shall concentrate on the idea that most attenuation will happen very close to the GC source.

3. Given the similarities in the slopes of the fitted power laws shown in figure 1, the attenuation/degradation should be energy independent over the observed TeV data points. At the heuristic level, at least, pair-production can achieve something like this (though this is somewhat dependent on the distribution of the target photon population) once one accounts for the possibility that the daughter electron-positron 
pairs go on to produce further, high energy $\gamma$-rays by inverse Compton scattering of light in the background radiation field, thereby initiating a cascading process which redistributes the photon energy ${ }^{8}$.

4. The main remaining question now is simply whether we can plausibly get a sufficient column density of NIR-optical photons near a candidate GC source to effect the attenuation/degradation. For reasons that are explained in $\S 8$ the two objects we consider plausible sources for the EHE cosmic rays are the accretion disk associated with the GC black hole itself, Sgr $\mathrm{A}^{*}$ and a supernova remnant located very close to the GC called Sgr A East. We consider the photon environments in the immediate vicinity of each of these before briefly considering the general, GC photon number density (i.e, within $\sim 10$ pc of the center).

(a) Sgr A*: Genzel et al. (2003) find that the "local background subtracted" luminosity of Sgr $\mathrm{A}^{*}$ in the NIR is $\sim 3.8 \times 10^{34} \mathrm{erg} . \mathrm{s}^{-1}$. This means - assuming a point source - a column density along a radial direction starting at $r_{0}$ is given by $2.7 \times 10^{34}\left(r_{0} / \mathrm{cm}\right)^{-1} \mathrm{~cm}^{2}$. Setting this equal to the required column density $\left(3 \times 10^{25}\right.$ $\mathrm{cm}^{2}$ ) and inverting we find $r_{0} \sim 10^{9} \mathrm{~cm}$. But this is inside the Schwarzschild radius of the central black hole $\left(\sim 8 \times 10^{11} \mathrm{~cm}\right)$ and, therefore, an unphysical requirement. The NIR light field due to the Sgr A* accretion disk, in other words, cannot attenuate the $\mathrm{TeV} \gamma$-rays to the extent we require. We note in passing that postulating a source location very close to the central black hole would present many observational difficulties. These are summarised in $\S 8.1$

(b) Sgr A East: From fig 3 of Melia et al. (1998) the extrapolated synchrotron flux at $\sim 1 \mathrm{eV}$ for this object is $0.3 \mathrm{MeV} \mathrm{cm}{ }^{-2} \mathrm{~s}^{-1} \mathrm{MeV}^{-1}$. This translates to a rough (number) luminosity of $2.6 \times 10^{45}$ photons $\mathrm{s}^{-1}$, which is an order of magnitude smaller than that for Sgr A* which means an analogous $r_{0}$ many orders of magnitude too small given the $\sim$ pc scales of the Sgr A East shock(s).

(c) General background: On the other hand, the actual quantity of interest is not the background-subtracted luminosity nor the luminosity due to any particular object. Rather, it is the total number density of suitable photon targets in the GC environment. This we can estimate from the following consideration: Wolfire,

\footnotetext{
${ }^{8}$ In this regard, consider figures $6 \mathrm{a}$ and $6 \mathrm{~b}$ of Carraminana (1992), which show the results of detailed modeling of the "self-attenuation" of $\mathrm{TeV} \gamma$-rays from two X-ray binary systems due to interactions with thermal NIR and optical photons emitted by those systems' own accretion disks. It can be seen in these figures that the resultant (down-shifted) spectrum parallels the unmodified spectrum for up to an order of magnitude in energy.
} 
Tielens and Hollenbach (1990) find that the GC circumnuclear disk requires an ionizing UV photon flux of 100-1000 erg $\mathrm{cm}^{-2} \mathrm{~s}^{-1}$. Taking the upper figure and assuming the same energy density in NIR photons ${ }^{9}$ one finds (at $\sim 1 \mathrm{eV}$ ) a photon number density of $2.1 \times 10^{4} \mathrm{~cm}^{-3}$. Again, given the specified column density, this number density requires a length scale of $1.5 \times 10^{21} \mathrm{~cm} \sim 500 \mathrm{pc}$ to get sufficient attenuation. This would seem to be excessive.

We reluctantly conclude, then, that around neither of the plausible, GC sources of the EHE cosmic rays, nor in the general GC environment, does one find a large enough NIRoptical photon number density (over sufficient scales) for our puposes: the optical depth to pair production experienced by the $\mathrm{TeV} \gamma$-rays in their propagation out of the GC environment is too small for us to explain the totality of data with a single source. We now consider, therefore, the idea that two effective sources are contributing to the totality of data with one source explaining the EGRET results and another (hopefully) able to account for both the HESS and EHE cosmic ray observations. In this scenario we do not have a compelling explanation for the closely parallel nature of the two fitted lines in figure 1 aside from the general expectation from shock acceleration theory that the spectral index be close to 2.0 in a strong shock.

\subsection{Two Effective Sources?}

In introducing the idea that there may be two effective ${ }^{10}$ sources we note that this is not an entirely unnatural reading of the situation. There are two pieces of evidence we bring in here:

1. It has actually been determined by Hooper and Dingus (2002) in their re-analysis of select data from the the $3 \mathrm{EG}$ catalog (Hartman et al. 1999) that the GC is excluded at the $99.9 \%$ confidence limit as the true position of the source 3EG J1746-2851 (this determination is at variance with the findings made in the Hartman et al. (1999) paper). Hooper and Dingus found the EGRET source to be fairly well localised to

\footnotetext{
${ }^{9} \mathrm{~A}$ re-processed IR photon background of similar energy density to the $30000 \mathrm{~K} \mathrm{UV}$ background is, in fact, expected, but this would realistically peak at around $100 \mathrm{~K} \sim 2.3 \times 10^{-2} \mathrm{eV}$ : see $\S 6.3 .1$. Given that, in any case, we do not find any positive effect from the re-processed IR photons this detail need not concern us.

${ }^{10}$ We emphasise 'effective' here because two or more apparent sources might in fact orginate from a background population of protons accelerated in different regions of a single, extended object.
} 
a position $0.21^{\circ}$ south west of the $\mathrm{GC}$ (i.e., $\sim 5$ pc at this distance). In contrast, the HESS GC source was found to lie with $95 \%$ confidence within $0.05^{\circ}$ of the GC (Aharonian et al. 2004).

2. Both modeling and observation of SNRs over many years has consistently shown that those examples with high flux tend to have a lower energy cutoff than those whose spectrum extends to higher energies. This is generally attributed to the fact that physical conditions that sponsor efficient acceleration also lead to efficient cooling at higher energy. In general, then, the flux level and energy cutoff tend to go in opposite directions (see, e.g., Baring (1999)).

On the quantitative side, the first item we must now check is whether a good fit is possible to the combined HESS+EHECR data which, in this scenario, are supposed to be explained by a single source (we call this assumed high-energy source the 'HE source'). We find this, indeed, to be the case: such a fit produces a $\chi^{2}$ value of 4.6 and a reduced $\chi^{2}$ of 0.51 for the $(11-2)$ dof. The best fit spectral index is very hard: 1.97 . If we constrain the spectral to be 2.0 and fit only to the normalization we obtain a $\chi^{2}$ of 5.0 and a reduced $\chi^{2}$ of 0.50 for $(11-1)$ dof (see figure 2).

The second issue we must confront is whether the other source (which we label the 'LE source') - associated with the signal seen by EGRET - in any way interferes with the TeV observations which are ascribed to the HE source. In particular, we must determine whether the LE source is "overtaken" by the HE source at or below HESS energies as we require (in order that $\sim \mathrm{TeV} \gamma$-rays not be overproduced). This might happen in either (or in an effective combination of) two ways: (i) if the spectral slope of the LE source is sufficiently steeper than the HE source and/or (ii) if the LE source cuts out below TeV energies (the requirement that this source not produce a relatively significant flux of $300 \mathrm{GeV}$ and above $\gamma$-rays would be guaranteed if the parent protons cut out at or below $\sim \mathrm{TeV}$ ).

In regard to (i) and (ii) immediately above, we note firstly that (i) appears not to be the case: we have tried two approaches here and both overpredict the differential $\gamma$-ray fluxes at $\sim \mathrm{TeV}$ by at least an order of magnitude. In the first approach we fit only to the three (highest energy) EGRET data points for 3EG J1746-2851 with variable normalization and spectral index. This naive approach produces a very steep spectral index of 2.6. In the second approach we fit only to normalization fixing the spectral index to 2.4 which is at the upper limit of the spectral index range for the parent proton spectrum as determined by Fatuzzo and Melia (2003) in their fit to the totality of the EGRET data (i.e., all 9 data points).

We should consider, then, whether (ii) could describe the situation. 
The interesting question now is, therefore, how we arrange for the difference of something like seven orders of magnitude between the maximum energies attained in the LE and HE GC sources. Certainly variation in ambient particle densities and magnetic field strengths will go some way towards explaining the difference, but it is doubtful that the combination of these two could give a difference of $10^{7}$. Another tenable hypothesis is that the LE source - which in this scenario would be entirely independent of the HE source - has an age-limited maximum energy. Another scenario would posit that the lion's share of the difference may be attributed to differences in shock geometries. In particular, one could postulate that the HE source realize a perpendicular shock configuration and that the LE source be described by a parallel configuration. This difference would be expected to contribute to at least two orders of magnitude variation in maximum acceleration energies (all other considerations aside): see $\S 7$, in particular, equations (38) and (39) below. Note that we would also expect different shock compression ratios in the different effective sources so the generic expectation would be for differing spectral indices.

\subsection{Other Neutron Production Channels}

There are two other channels which might reasonably contribute to EHE neutron production at the GC, viz. p- $\gamma$ and heavy ion dissociation. Both of these operate, effectively, without producing a concomitant $\mathrm{GeV}$ or $\mathrm{TeV} \gamma$-ray signal. This has the implication that our calculation of EHE neutron production (normalized to this $\gamma$-ray signal) is, all other things being equal, a strict under-estimate.

\subsection{1. $p \gamma$ interactions}

We have performed detailed calculations of the $\mathrm{p} \gamma$ process within the $\Delta(1232)$ resonance approximation (Stecker 1979; Mucke et al. 1999; Dermer 2002). In this context, the relevant neutron production channel is through the first $\Delta$ resonance at $1232 \mathrm{GeV}$,

$$
p \gamma \rightarrow \Delta \rightarrow n \pi^{+},
$$

with a cross-section of about $6 \times 10^{-28} \mathrm{~cm}^{2}$ (Hagiwara and al. 2002). Here the branching ratios of the $\Delta(1232)$ lead to proton to neutron production in the well-defined ratio of $2: 1$ and its decay kinematics predict a nucleon elasticity of 0.8 (Mucke et al. 1999). The interaction

can take place if the energy of the ambient photon in the p rest frame, $E_{\gamma(p)}^{\operatorname{thresh}(\text { pion })}$, satisfies

$$
E_{\gamma(p)}^{\text {thresh(pion })} \geq \frac{m_{\Delta}^{2}-m_{p}^{2}}{2 m_{p}} \simeq 340 \mathrm{MeV} .
$$


This means that the relevant target photon population in the GC context is supplied by the intense flux of IR photons from the circumnuclear disk. This is a powerful source $\left(\simeq 10^{7} L_{\odot}\right)$ of re-processed mid- to far-infrared continuum emission with a dust temperature of $\simeq 100$ $\mathrm{K}$ (Telesco 1996). Given this temperature, we find that the $\mathrm{p} \gamma$ process does not contribute significantly for neutron production until proton energies of $\geq 6.9 \times 10^{18} \mathrm{eV}$ are reached, or, given the elasticity, does not contribute significantly to neutrons with energies below $\sim 5.5 \times 10^{18} \mathrm{eV}$. (The fact that this reaction does not 'kick-in' until such high energies explains why it can not be directly normalized to the $\mathrm{GeV} \gamma$-ray signal: despite the fact that the decay of neutral pions from the other branch of the $\Delta$ decay will certainly produce photons, these will all be directly produced in the EHE regime.) This fact - our detailed modeling shows - means that the $\mathrm{p} \gamma$ process produces a EHE neutron flux in the relevant energy range, at most, $\mathcal{O}[1 \%]$ of that due to pp and may, therefore, be ignored.

Note that we have performed the calculations in this section assuming that the interaction region is at $\mathcal{O}[\mathrm{pc}]$ scales from the GC (appropriate, e.g., to the closer-in parts of the Sgr A East shell). We explain in $\S 8.1$ why it is unlikely that the interaction region be very much closer to the GC than this

\subsubsection{Heavy Ion Dissociation}

A calculation of neutron production from dissociation of heavy ions (through interactions with either ambient protons or light) is beyond the scope of this paper (Tkaczyk 1994). Such interactions, moreover, do not lead directly to photon production at any energy and we can not, therefore, normalize the rate of this process to the EGRET $\gamma$-ray data directly. Still, that this mechanism might be operating we find entirely plausible especially given the fact that heavy ions make up a non-negligible fraction of the detected cosmic ray population.

\section{Limiting Energies}

As remarked above, the flux figures listed in Table 3 implicitly assume that the powerlaw description of the parent protons which (indirectly) generate the photons observed at $\sim 5 \times 10^{9} \mathrm{eV}$ continues to hold up to much higher energies, $10^{18} \mathrm{eV}$ and above. It also assumes that the distribution of daughter neutrons continues to be set by the scaling relation described in equation (16). This latter point we discuss in detail in an appendix. In brief, 
we expect that the scaling relation will be correct to within a factor of two ${ }^{11}$, which, given other uncertainties in the calculation, does not introduce a significant extra uncertainty in our neutron flux calculations. Certainly, however, we need to devote considerable attention to the former point regarding the maximum energies to which any particular known source at the GC might accelerate protons. We shall see that considerations surrounding limiting energy actually mean that we can - with some confidence - identify which of the potential sources at the GC is responsible both for the EGRET-observed signal and the neutron signal.

In determing the limiting energy, $E_{p}^{\max }$, two broad considerations play a part, viz (i) the intrinsic limits to $E_{p}^{\max }$ given the macroscopic properties of the shock at the source doing the acceleration (magnetic field strength, shock size, geometry, and age, etc) and (ii) limits to $E_{p}$ from heating-cooling balance which we label scattering limits.

We discuss both (i) and (ii) at greater length below.

\subsection{Perpendicular Shock Acceleration}

\subsubsection{Transport}

The whole of diffusive shock acceleration theory can be obtained from the Parker equation, the fundamental transport equation for the charged particle distribution function, $f(\vec{r}, t, p)$, in a background, collisionless hydromagnetic field (Parker 1965):

$$
\frac{\partial f}{\partial t}=\frac{\partial}{\partial x_{i}}\left[\kappa_{i j} \frac{\partial f}{\partial x_{j}}\right]-U_{i} \frac{\partial f}{\partial x_{i}}+\frac{1}{3} \frac{\partial U_{i}}{\partial x_{i}} \frac{\partial f}{\partial \ln (p)}+Q\left(x_{i}, t, p\right),
$$

where $\kappa_{i j}$ is the diffusion tensor and $Q\left(x_{i}, t, p\right)$ is the local source strength. This equation parametrizes the effects of diffusion, convection, and acceleration/deceleration by an electric field on the charged particle distribution. The equation assumes that the diffusion approximation holds good, i.e., that particles are scattered often enough by magnetic field irregularities that the particle distribution is nearly isotropic. The Parker equation also requires that the ratio of the shock speed, $U=|\mathbf{U}|$, to the particle speed, $w$ is small: $U / w \ll 1$. In this picture, particle acceleration is caused by two factors (Jokipii 1982, 1987):

1. the large relative motion between the (magnetic) scattering centers (i) causing the diffusion in front of the shock and (ii) behind the shock

\footnotetext{
${ }^{11}$ Paolo Lipari, private communication, 2003.
} 
2. (if the magnetic field has a component normal to the direction of propagation of the shock) drift along the shock front.

Despite the potential importance of the second factor listed above, many discussions neglect magnetic field changes and the resulting particle drifts, effectively restricting consideration to quasi-parallel shocks.

From observation, it is known that the Galactic cosmic ray flux is quasi-isotropic to high energy. This indicates that the Parker equation should hold as a valid description of cosmic ray acceleration. The scattering of a charged particle depends most strongly on the amplitude of magnetic turbulence on scales similar to that particle's gyroradius. This consideration justifies the 'quasi-linear' approximation in which the scattering rate is taken to be proportional to the fluctuation spectrum of the magnetic field at a wavenumber approximately equal to the inverse of the gyroradius.

\subsubsection{Acceleration}

Charged particle acceleration in the collisionless plasmas of space is through the action of ambient electric fields, E. Because the relevant plasma processes are hydromagnetic (i.e., because we expect the charges to respond to changes in the electromagnetic field on a time scale much shorter than the flow time, so that the Lorentz force in the plasma frame is effectively zero) the electric field satisfies

$$
\mathbf{E}=-\mathbf{U} \times \mathbf{B}
$$

Note that though $\mathbf{E}$ does not appear explicitly in the Parker equation, it is implicitly contained in $\mathbf{U}$. We can arrive at a completely general description of diffusive shock acceleration (i.e., applying equally well to parallel and perpendicular shocks and everything in between) by putting a step-function $\mathbf{U}$ into the Parker equation.

In the strong shock limit, $r$, the ratio between the upstream and downstream flow velocities satisfies

$$
r=\frac{U_{1}}{U_{2}} \rightarrow 4
$$

(note that a subsript of 1 indicates a quantity measured upstream or in front of the shock, whereas a subscript 2 indicates a quantity downstream or behind the shock). In this case, it can be shown that the steady-state solution of the Parker equation indicates that the momentum dependence of the particle distribution goes like $f(p) \propto p^{-4}$ corresponding to an energy spectrum $p^{2} f \propto p^{-2}$. This describes the universal power law (in either momentum or 
energy) of spectral index close to 2.0 expected at strong shocks, whatever the particulars of shock speed, diffusion coefficients, etc.

\subsubsection{Acceleration Rate}

In contrast to the spectral index, the high energy cut-off of the accelerated particles is sensitive to the particulars of the shock. This maximum energy is largely determined by the time available to accelerate the particles and the rate of energy gain. These, in turn, are controlled by the finite lifetime of the shock itself, the escape of particles from the shock region, and the rate at which particles scatter back and forth across the shock (collision and synchrotron losses are also important, but these are dealt with below).

By solving the time-dependent Parker equation (and taking particles to be injected into the shock at $t_{0}$ with momentum $p_{0}$ ), we can determine that the particle distribution is still governed by the universal power law previously determined but now with a high momentum cut-off, $p_{c}$, which satisfies

$$
\frac{\mathrm{d} p_{c}}{\mathrm{~d} t} \simeq U_{\text {shock }}^{2} \frac{p_{c}}{4 \kappa_{x x}}
$$

where $\kappa_{x x}$ is the diffusion coefficient normal to the shock front. Either increasing the shock speed, $U_{\text {shock}}$, or decreasing the diffusion coefficient, therefore, will produce a greater rate of maximum momentum increase and, consequently, lead to faster particle acceleration in general. Now, we can write the diffusion coefficient normal to the shock front as

$$
\kappa_{x x}=\kappa_{\|} \cos ^{2}\left(\theta_{B}\right)+\kappa_{\perp} \sin ^{2}\left(\theta_{B}\right)
$$

where $\theta_{B}$ is the angle between the shock normal and the magnetic field vector. One can see immediately, then, that, if the shock is quasi-perpendicular, $\kappa_{x x} \simeq \kappa_{\perp}$, because $\kappa_{\perp}$ is usually smaller than $\kappa_{\|}$, such a shock will tend to accelerate particles faster than a quasi-parallel shock.

Let us consider the two limiting cases, quasi-parallel and quasi-perpendicular shocks, in a little more detail.

\subsubsection{Quasi-parallel Shocks}

This is the more-commonly investigated case. From equation (31) we shall have that

$$
\frac{1}{p_{c}} \frac{\mathrm{d} p_{c}}{\mathrm{~d} t} \simeq \frac{U_{\text {shock }}^{2}}{4 \kappa_{\|}}=\frac{3 U_{\text {shock }}^{2}}{4 \lambda_{\|} w},
$$


where $\lambda_{\|}$is the mean free path along the shock. Now, given that this quantity must be of the order of or larger than the gyroradius of a particle at the limiting upper momentum, $r_{g}\left(p_{c}\right) \equiv r_{c}$, we find that

$$
\frac{1}{p_{c}} \frac{\mathrm{d} p_{c}}{\mathrm{~d} t} \lesssim \frac{3 U_{\text {shock }}^{2}}{4 r_{c} w}
$$

which is the so-called Bohm limit on the acceleration rate. Many researchers have taken this to represent a mechanism-independent limit on the acceleration rate and, hence, indirectly on $E_{\max }$. The logic implicit here is that in order to be turned about so that it re-crosses the shock, a particle must be re-scattered each time it moves up- or downstream of the shock. The highest energy gain rate, by this logic, will then occur for the smallest scattering mean free path, which, in turn, cannot be smaller than the gyroradius.

\subsubsection{Quasi-perpendicular Shocks}

The reasoning presented above does not have universal validity, however: if the magnetic field has a component perpendicular to the shock propagation direction and if (as is usually the case as mentioned above) $\kappa_{\|}>\kappa_{\perp}$ (so that the particle is less constrained in motion along the shock than in motion away from it), the gyromotion of a charged particle can carry it across the shock many times between each scattering. This can mean a much larger $E_{\max }$ than for the parallel case.

To see this quantitatively, note that the kinetic theory (i.e., billiard ball scattering) value for the ratio between perpendicular and parallel diffusion coefficients is given by

$$
\frac{\kappa_{\perp}}{\kappa_{\|}}=\frac{1}{1+\left(\lambda_{\|} / r_{g}\right)^{2}},
$$

so that for a larger parallel mean free path, the perpendicular diffusion coefficient is reduced and the acceleration is, therefore increased (to be contrasted with the parallel case for which a smaller parallel mean free path means an increase in the acceleration rate).

It seems that if we want acceleration to very high energies we should simply dial up the required $\lambda_{\|}$. This quantity may not be increased without limit, however. There will, in fact, be a maximum value for $\lambda_{\|}$which can be determined (interchangeably) by the logic that (i) the diffusion approximation implicit in the Parker equation ceases to be valid if particles do not scatter often enough to be isotropic at the shock or (ii) particles must scatter often enough to diffuse upstream fast enough to stay ahead of the shock (Jokipii 1982; Jokipii 1987) Either of these two conditions leads to the requirement that

$$
\Upsilon \equiv U_{\text {shock }} \frac{r_{g}}{\kappa_{\perp}} \ll 1
$$


or, for simple scattering

$$
\Upsilon \simeq \frac{\lambda_{\|}}{r_{g}} \frac{U_{\text {shock }}}{w} \ll 1,
$$

so that if $U_{\text {shock }} / w \ll 1$ then we can have $\lambda_{\|} / r_{g} \gg 1$ (while still maintaining Eq. 37), giving us a significant enhancement over the Bohm acceleration rate.

\subsubsection{Limiting Energies in Diffusive Shock Acceleration at Supernova Remnants}

Postulating that the maximum energy to which a Supernova Remnant (SNR) shock might accelerate particles, $E_{\max }^{\mathrm{SNR}}$ is given by (i) the Bohm value of the diffusion coefficient and (ii) the modified Sedov solution for $U_{\text {shock}}$, Lagage et al. (1983) determined that

$$
E_{\max }^{\mathrm{SNR}} \sim E_{\max }^{\mathrm{L} \& \mathrm{C}} \equiv \text { few } \times 10^{14} \times Z \mathrm{eV}
$$

for the distance and magnetic field scales typical for a Galactic SNR. Given that, as outlined above, SNRs are expected to accelerate the bulk of the cosmic rays up to at least the knee at $\sim 5 \times 10^{15} \mathrm{eV}$, equation (38) would seem to place a severe constraint on the mass composition of the cosmic rays.

Following from the arguments presented above, however, Lagage and Cesarky's result will not apply to quasi-perpendicular shocks. The sort of (globally) spherical shock produced by a supernova, moreover, is quasi-perpendicular over much of its area, meaning that $\kappa_{\perp}$ is much more important that $\kappa_{\|}$over a substantial fraction of the SNR shock and the acceleration rate can be much larger than the Bohm limit. This means $E_{\max }^{\mathrm{L} \& \mathrm{C}}$ is actually a significant underestimate of $E_{\max }^{\mathrm{SNR}}$ which, by putting $\Upsilon$ equal to its maximum value, viz. $\sim 0.3$, in equation $(37)$ we can determine to be

$$
E_{\mathrm{Z}}^{\max (\text { perp })} \sim 5 \times 10^{16} \times Z\left(\frac{B}{20 \mu G}\right)\left(\frac{R_{\text {shock }}}{10 \mathrm{pc}}\right) \mathrm{eV}
$$

for a particle or nucleus of charge $\mathrm{Z}$ (see Appendix $\mathrm{C}$ for a full derivation of the limiting energy). Note that in the equation above "perp" signifies that the maximum energy appertains to acceleration in a perpendicular shock geometry (Jokipii 1982; Jokipii 1987). This energy is amply large enough to accelerate cosmic rays beyond the knee but note that equation (39) represents an in-principle limit: in general, cooling effects due to synchrotron radiation or collisions between the accelerated protons (and ions) and ambient matter or light may more tightly limit the maximum energy/momentum to which the high energy particle population

can be accelerated. Further, the time available for acceleration is, of course, bound by the total age of the shock. We now discuss these additional constraints. 


\subsection{Cooling- and Age-Imposed Limits to Maximum Particle Energies}

There will, in general, be a maximum proton energy $E_{p}^{\max }$ above which the combined energy loss rate due to many processes - proton synchrotron emission, inverse Compton scattering and hadronic collisions (with ambient nucleons and $\gamma$ 's) - will exceed the rate of energy gain due to shock acceleration.

In the GC environment, however, pp collisions are, by far, the dominant energy-loss process (Markoff et al. 1997; Melia et al. 1998; Fatuzzo and Melia 2003). This is the case becasue of the relatively lower density of target photons and the intrinsically smaller cross-sections and inelasticities of $p \gamma$ processes in comparison with pp collisions (this is to be contrasted with the situation that generally pertains in AGN, where the photon density can be significantly higher than in the GC).

This maximum energy, $E_{p}^{\max (\mathrm{cool})}$, attainable given that pp collisions are occuring, depends on the functional form of the average collision inelasticity, where inelasticity denotes the fractional energy loss in the collision (i.e., the energy of the leading baryon in units of the energy of the incoming proton). The time scale for proton cooling via pp collisions, then, is given by

$$
t_{p p} \simeq \frac{1}{n_{p} c \sigma_{p p} K_{p p}}
$$

where the proton-proton cross-section, $\sigma_{p p}$, and the fractional energy loss per pp collision or inelasticity, $K_{p p}$, are both, in principle dependent on energy (see the appendix).

On the other hand, the proton (shock) acceleration time scale is given by (Begelman 1990)

$$
t_{p}^{\mathrm{acc}}\left(E_{p}\right)=\frac{E_{p}}{\eta c^{2} e B}
$$

where $\eta$ is a dimensionless parameter, $\mathcal{O}[1]$, that depends on the details of the acceleration mechanism.

We can determine $E_{p}^{\max }$, then, by setting

$$
t_{p p}=t_{p}^{\mathrm{acc}}\left(E_{p}^{\max }\right)
$$

and inverting to find

$$
E_{p}^{\max (\mathrm{cool})}=\frac{c \eta e B}{n_{p} \sigma_{p p} K_{p p}} .
$$

Finally in regard to maximum energies, one must also check that the time-scale implied by equation (41) for the maximum energy calculated using equation (43) does not exceed 
the age, $t_{\text {age }}$, of the pertinent astrophysical object or environment. If this is the case, the maximum energy will be age limited, i.e.,

$$
E_{p}^{\max (\text { time })}=\eta c^{2} e B t_{\text {age }} \simeq 2.4 \times 10^{18}\left(\frac{B}{20 \mu G}\right)\left(\frac{t_{\text {age }}}{2010^{4} \mathrm{yr}}\right) \mathrm{eV} .
$$

In general we shall have, then,

$$
E_{p}^{\max }=\min \left\{E_{p}^{\max (\mathrm{perp})}, E_{p}^{\max (\mathrm{cool})}, E_{p}^{\max (\text { time })}\right\}
$$

where the maximum energies are given, respectively, by equations (39), (43), and (44).

\section{Identifying the GC Neutron Source}

\subsection{Sgr $\mathrm{A}^{*}$ as EHE GC Neutron Source}

The question of the maximum energy to which protons might be accelerated at the Sgr A* shock has been addressed at some length by Markoff et al. (1999) and here we simply quote their results. As presaged above, these authors found that pp collisions are the dominant energy loss process for relativistic protons: $p \gamma$ processes are supressed relative to the expectation from other galactic nuclei due to the very low luminosity of the GC and $\mathrm{pe}^{-}$ processes are also suppressed due to a dearth of extremely energetic electrons. Taking the mass of the central black hole to be $2.61 \pm 0.65 \times 10^{6} M_{\odot}\left(\right.$ Eckart and Genzel 1997) ${ }^{12}$, the shock to be located at 40 Schwarzschild radii, a (generously large) magnetic field of $\simeq 300 \mathrm{G}$, and, finally, an ambient proton density of $\simeq 2 \times 10^{8} \mathrm{~cm}^{-3}$, Markoff et al. (1999) determine that the maximum attainable Lorentz factor for relativitic protons is approximately $4 \times 10^{8}$ which translates to $E_{p}^{\max } \simeq 4 \times 10^{17} \mathrm{eV}$. This maximum energy, then, means that Sgr $\mathrm{A}^{*}$ is probably not the source of the putative EHE neutrons seen by AGASA and SUGAR (indeed, even if the anisotropy is due to the direct interactions of the primary,accelerated protons, it still cannot be the source).

Another factor supporting the view that Sgr A* cannot be the source of the observed $\mathrm{EeV}$ neutron flux would be the inconsistency implied by the emissivity of secondary particles. The ensuing particle cascade would produce a copious supply of energetic electrons and positrons that, in the presence of the inferred $\sim 1-10$ Gauss magnetic field (let alone the $300 \mathrm{G}$

\footnotetext{
${ }^{12}$ Note that a recent revision to the Galactic center black hole mass that determines it to be $4.1 \pm 0.6 M_{\odot}$ (Ghez et al. 2003) does not substantially alter the conclusion of Markoff et al. (1999) regarding maximum attainable energies for shocked protons.
} 
field we used for the estimate above) for this source, would greatly exceed Sgr A*'s observed radio flux. This problem is alleviated if instead the secondary particles gyrate in the much weaker field associated with a typical supernova shell. Finally, Kosack et al. (2004) make the point that the lack of variability seen in their $\sim \mathrm{TeV} \gamma$-data for the GC source tells against the identification of this source with a compact point source such as Sgr A* (no evidence for variability is seen in the EGRET data for 3EG J1746-2851 either: Mayer-Hasselwander et al. 1998).

\subsection{Sgr A East as EHE GC Neutron Source}

\subsubsection{Introduction to Sgr A East}

Sgr A East is a mixed-morphology, $\mathcal{O}\left[10^{4}\right]$-year-old supernova remnant (SNR) located within several parsecs of the Galactic Center (GC) (see Melia and Falcke 2001). It appears to be of the class of SNRs which have been observed and detected at $1720 \mathrm{MHz}$ (the transition energy of $\mathrm{OH}$ maser emission), such emission being a signature of shocks produced at the interface between supersonic outflow and the dense molecular cloud environments with which these SNRs are known to be interacting. There is, further, good evidence (see below) that Sgr A East may be associated with the EGRET source 3EG J1746-2851 introduced in $\S 4$. It should be remarked, however, that if this putative association is real, Sgr A East has a $\gamma$-ray luminosity $\left(\sim 2 \times 10^{37} \mathrm{erg} \mathrm{s}^{-1}\right)$ almost two orders of magnitude greater than that of the other EGRET-detected SNRs, something which clearly demands explanation.

In fact, in a self-consistent scenario explored elsewhere (Melia et al. 1998; Fatuzzo and Melia 2003) the Sgr A East remnant has been shown to be capable of producing the observed $\gamma$-ray luminosity once the unusually-high ambient particle density $\left(\gtrsim 10^{3} \mathrm{~cm}^{-3}\right)$ and strong magnetic field ( $\gtrsim 0.1-0.2 \mathrm{mG}$ ) at the $\mathrm{GC}$ are taken into account. Indeed, let us make the natural assumption that there is a population of protons at Sgr A East accelerated by the shocks associated with the supernova blast wave (and, therefore, governed by a power law with spectral index $\sim 2$ ). Then, from collisions between these high energy protons and ambient ions, pions will be produced and these will, in turn, decay to produce $\gamma$ 's. Now, the high-energy $\gamma$-ray luminosity in this scenario is essentially dependent on the rate of collisions between ambient ions and high energy protons, which is, in turn, dependent on the product of the ambient ion density and the high energy proton density, $n_{\mathrm{H}} n_{0}$. So, we can fit to these two on the basis of the observed $\gamma$-ray spectrum. We also have, however, a handle on $n_{\mathrm{H}}$ from observations by ACIS on board the Chandra X-ray Observatory - Sgr A East is expanding into an ionized gas halo with a density $\sim 10^{3} \mathrm{~cm}^{-3}$ and interacting with a cloud

of density $\sim 10^{5} \mathrm{~cm}^{-3}$. Further, the total energy in the accelerated proton population is 
readily calculated from $n_{0}$ and is, of course, constrained to be less than the total energy released in the original explosion that gave birth to the Sgr A East shell.

These considerations result in fairly rigorous contraints on any attempted fit to observations of Sgr A East. But, with a proper treatment of pion production (in particular, the energy dependence of the pion multiplicity), the entire broadband spectrum of the object can be compellingly explained. This explanation essentially sees the super-100 MeV $\gamma$-ray spectum as due to $\pi^{0}$ decay, the sub-100 MeV $\gamma$-ray spectum explained by bremsstrahlung emission (self-consistently with the assumed value for $n_{\mathrm{H}}$, namely $10^{3} \mathrm{~cm}^{-3}$ : see below) and the VLA observations of Sgr A East at 6 and $20 \mathrm{~cm}$ explained by synchrotron emission (though see below for a caveat regarding the radio spectrum). Note that in this scenario, it is the secondary, charged leptons (ultimately resulting from collisions of the shock accelerated protons with ambient ions) that are responsible for the bremsstrahlung and synchrotron emission, rather than directly accelerated leptons. Indeed, the population of this primary lepton class can, in fact, be shown to be negligible in comparison with the secondaries, given that each accelerated proton produces of order 20-30 charged leptons (Fatuzzo and Melia 2003).

In regard to Sgr A East's radio spectrum, it should be noted that the synchrotron emission from the total population of secondary, charged leptons is not directly consistent with radio observations: the observed spectral index is $\sim 1$, pointing to an underlying population of non-thermal charged leptons with a power-law distribution of index $\sim 3$ (Pedlar et al. 1989). In contrast, from general considerations (whether the lepton population is directly accelerated or born of the interactions of shock accelerated protons), we expect that the lepton index be $\sim 2$, leading to radio emission characterized by a spectral index $\sim 0.5$. If, however, one allows for the reasonable eventuality that high energy leptons diffuse out of the Sgr A East region, the convolution of a diffusion loss factor with the initial lepton spectrum resulting from pion decay allows a very good fit to the radio spectrum (Fatuzzo and Melia 2003). Alternatively, it might be that the high energy protons themselves are diffusing out of the system thus also (indirectly) depleting the high-energy lepton spectrum. This scenario also provides a credible, though less good, fit to the radio data. As will become clear, however, the very fact that we do see a GC EHE cosmic-ray anisotropy argues against the latter scenario.

The scenario presented above - which we label 'HIGH' - is to be contrasted with an alternative picture ('LOW') explored earlier (Melia et al. 1998), in which a considerably lower ambient particle density was employed (viz. $\sim 10 \mathrm{~cm}^{-3}$ ). Through the $n_{\mathrm{H}} n_{0}$ dependence of the $\gamma$-ray luminosity this then necessitated a large population of high energy protons, in turn yielding an energy content of relatitivistic particles of $\sim 8 \times 10^{50} \mathrm{erg}$. In contrast, the particle energy calculated for the HIGH scenario is $\sim 10^{49}$ erg so clearly the view that 
Sgr A East resulted from a standard supernova favors HIGH. Moreover, the recent Chandra observations of Sgr A Eastalluded to above support the view that Sgr A East is, indeed, the remnant of a single supernova with typical energetics.

\subsubsection{Maximum Energy of Protons Accelerated by the Sgr A East Shock}

Typically, the magnetic field strength in the interstellar medium scales as the square root of the density. In the case of Sgr A East, it appears that most of the pp scatterings (and essentially all of the bremsstrahlung and synchrotron emissivity) occur in the $\sim 10^{3} \mathrm{~cm}^{-3}$ region, where the field strength is $\sim 0.2 \mathrm{mG}$. Thus, if the shock occurs in the $\sim 10^{5} \mathrm{~cm}^{-3}$ molecular cloud colliding with the expanding shell, the field intensity within the particle acceleration region may be as high as a few $\mathrm{mG}$. However, a value greater than this seems implausible.

With a field strength of $4 \mathrm{mG}$, the other inputs described above, and the application of equation (39), one finds that particles in the Sgr A East shock can be accelerated, in principle, to $10^{19} R_{\text {shock }} / 10 \mathrm{pc} \mathrm{Z} \mathrm{eV}$. This figure is not limited by cooling or age considerations: from equation (43) we find that, given the assumed $3,000 \mathrm{~cm}^{-3}$ ambient proton density ${ }^{13}$, a lower limit on the cooling-limited maximum energy is $2.7 \eta 10^{21} \mathrm{eV}$ (this is a lower limit because it has been derived assuming a pp cross-section of $150 \mathrm{mb}$, which, in fact, only pertains at the very highest region of the pertinent energy range). Moreover, the time required by the $4 \mathrm{mG}$ field to accelerate protons to this energy is around 2500 years, comfortably inside the $\mathcal{O}\left[10^{4}\right]$ year age of the remnant (the age-limited energy using equation (44) is around $\eta 5 \times 10^{20} \mathrm{eV}$ for a $4 \mathrm{mG}$ field and an age of 10000 years).

Parenthetically, note from the above that heavier ions might be accelerated to energies approaching $10^{20}$ at Sgr A East - potentially explaining most of the cosmic ray spectrum even above the ankle. If this were the case, it would not imply an unambiguous anisotropy towards the GC: even at $10^{19} \mathrm{eV}$ modeling shows that a proton leaving the vicinity of the GC would be scattered many times, on average, before it could reach us at Earth. This mechanism would isotropise the GC signal even at these extreme energies, though they would probably not completely wash it out. Such a scenario is not ruled out by current observations.

\footnotetext{
${ }^{13} \mathrm{As}$ is the case at $\mathrm{Sgr} \mathrm{A}^{*}$, pp collisions are the dominant cooling mechanism for relativistic protons accelerated at the Sgr A East shock (Melia et al. 1998; Fatuzzo and Melia 2003).
} 


\subsubsection{Sgr A Eastand the Two Source Model}

We note finally that there being two effective sources required to explain the totality of data fits naturally into the hypothesis that the Sgr A East shell accelerates all the protons required to explain the various signals. The Sgr A East shell ranges in distance from very close to the GC (within $1 \mathrm{pc}$ ) to up to $\sim 10 \mathrm{pc}$. It subtends, therefore, regions of widely varying ambient particle density and also magnetic field strength and orientation so that proton populations accelerated in it would be expected to display or experience varying maximum energies, spectral indices and overall (energy) differential number densities and interaction rates. Moreover, Hooper and Dingus's (2002) determination that the EGRET source can be localized to a region $0.21^{\circ}$ (i.e., $\sim 5 \mathrm{pc}$ ) away from the actual GC (while the HESS source is localized to within 1 pc of the GC) supports this basic idea: the lower energy source is located in a region where ambient magnetic fields can be expected to be lower and also, perhaps, where ambient particle densities be higher, whereas the higher energy source is located in a region of larger magnetic field strength. We hypothesise also that the acceleration in these regions be described by different shock/magnetic field geometries (with the EGRET source describing a parallel shock-magnetic field configuration and the HESS/cosmic ray source being described by the more efficiently-accelerating perpendicular configuration). Further observations and modeling will be required to determine whether this picture is tenable.

\section{Conclusion}

We have examined - and shown to be quantitatively successful - the idea that the Galactic center EGRET source 3EG J1746-2851 is also responsible for the observed anisotropy that is seen in cosmic rays towards the GC at an energy of around $10^{18} \mathrm{eV}$. We have, in particular, demonstrated that a power-law fit to the $\sim$ GeV EGRET data and the EHE cosmic ray anisotropy data (taking the latter to be evidence for neutron primary particles) requires a parent proton population with a spectral index of 2.23 . This is in exact agreement with previous determinations made (individually) for the spectral indices of the parent proton population that is generating the EGRET-observed $\gamma$-rays and for the distribution of the EHE cosmic ray signal. The determined spectral index is also in perfect agreement with the expectation from shock acceleration theory. Our fitting procedure requires input from the particle physics of charge-exchange in pp collisions. Our results imply the existence of a population of shock-accelerated protons (and perhaps heavier ions) at the GC with energies that range up to, at least, $\sim 10^{19} \mathrm{eV}$.

Unfortunately, however, this fit overpredicts the flux of $\gamma$-rays from the GC at $\sim \mathrm{TeV}$ 
energies given the existing data from a number of atmospheric Cerenkov telescopes (which are, admittedly, somewhat at variance amongst themselves). We show that what we believe to be the best and most recent data - that coming from the HESS instrument - is well fitted by a power law with a spectral index of 2.22, i.e., very close to the power law fit to the combined EGRET and EHE cosmic ray data. One explanation, then, of the deficiency of the $\sim \mathrm{TeV}$ data with respect to expectation is that the HESS instrument's normalization is wrong by a large (energy-independent) factor $(\sim 20)$. Finding this somewhat unlikely, however, we have sought astrophysical explanations of the totality of EGRET + HESS + EHECR data, taking these all to be fundamentally sound. Pursuing the idea that all the data might be explained as originating from the interactions of a single high energy proton population, we searched for mechanisms that would (preferentially) attenuate the $\sim \mathrm{TeV}$ $\gamma$-rays. The most promising candidate in this direction, however, viz, pair production on an assumed $1.5 \mathrm{eV}$ light background, we have found is unlikely to be efficacious as the number density of such photons in and around candidate GC sources or source regions is probably too low.

Noting these facts, we sought for a model involving two effective sources. We showed here that the hypothesis that the EGRET data are explained by one source and the HESS + EHECR data by another is perfectly tenable with, in particular, a good fit possible to the combined HESS + EHECR data. This two source scenario - which has some plausibility given the varying source position determinations made on the basis of observations conducted in the various energy regimes - requires that the EGRET source probably be steeper than - and certainly have a cut-off energy much lower than - the HESS + EHECR source.

This difference in maximum particle energies could be attributed to either completely independent populations of high energy protons, accelerated in different astrophysical sources with differing ages, magnetic field strengths, ambient particle densities, etc. Alternatively - and more plausibly in our opinion - a population of high energy protons accelerated in different regions (with differing magnetic field/shock geometries) of a single, extended object might produce two (or more) effective sources.

We have shown that the Sgr A East supernova remnant (located near the GC) could provide the environment in which the acceleration of the high energy protons we require could plausibly be achieved. Further, we have shown that the 'two-source' model fits naturally with the hypothesis that Sgr A East is the accelerated proton source: the Sgr A East shell ranges in distance from very close to the GC (within $1 \mathrm{pc}$ ) to up to $10 \mathrm{pc}$ distance. It subtends, therefore, regions of widely varying ambient particle density and magnetic field strength and orientation so that proton populations accelerated in it would be expected to display or experience varying maximum energies, spectral indices and overall (energy) differential 
number densities and interaction rates. On the other hand, it seems that the other plausible GC source, namely the accretion disk around the GC black hole, Sgr A*, is unlikely to provide an environment in which acceleration of protons to the extreme energies required is possible. Further, observations tell against a point-like source for both the $\mathrm{GeV}$ and $\mathrm{TeV}$ regime $\gamma$-rays. Our results, then, provide tentative evidence for the first direct connection between a particular, Galactic object (Sgr A East) and the acceleration of cosmic rays up to energies near the ankle in the cosmic ray spectrum.

At all energies, we anticipate - with great interest - the results that shall be obtained by the next generation of instruments. These instruments include the GLAST Large Area Telescope (Thompson 2004) at $\sim$ GeV energies and VERITAS (Krennrich et al. 2004), at $\sim \mathrm{TeV}$ energies, and, finally, the Pierre Auger Observatory (Camin 2004) for cosmic rays of $10^{18} \mathrm{eV}$ and higher. The results from these instruments will either confirm or rule-out our scenario.

\section{Acknowledgments}

RMC gratefully acknowledges useful communications from Paolo Lipari and enlightening conversations with Matt Holman, Jasmina Lazendic, Avi Loeb, and Josh Winn. FM would like to thank Steve Fegan and Trevor Weekes for very helpful correspondence. This research was supported in part by NASA grant NAG5-9205 and NSF grant 0402502 at the University of Arizona. RRV and FM are also partially supported by a joint Linkage-International grant from the Australian Research Council. RRV thanks Paolo Lipari and Todor Stanev for general discussions on cosmic rays and the observed anisotropies, and he thanks Paolo Lipari and Maxim Khlopov for their kind hospitality at the Universitá di Roma "La Sapienza" where these discussions took place. MF is supported by the Hauck Foundation through Xavier University.

\section{Appendix: Neutron Production in UHE Hadronic Interactions}

We discuss here the necessary, particle physics inputs to our calculation of neutron production, viz. the total (inelastic) pp cross-section, the leading neutron multiplicity in pp interactions and the leading neutron elasticity in pp. In summary of the below, all three of these quantities might in principle exhibit a scaling-violating dependence on the center of

mass energy. The data on the EHE cross-section are fairly sound and do indicate exactly such a dependence, albeit a weak one. We account for cross-section growth as set out in $§ 5.2$. 
There is no data at EHE on neutron multiplicity or inelasticity and, indeed, even at moderate energies the data are scant. Modeling, however, predicts little or no energy dependence in these quantities (note that we are talking about the production of leading particles, not secondaries). We take it, then, that the leading neutron multiplicity and elasticity do not evolve from the values supplied by the low energy data.

\subsection{Leading Neutrons from pp Collisions: General Remarks}

By definition, leading baryons (proton, neutron, or hyperon) created in pp (or pA) collisions are those particles that carry a large fraction of the incoming hadron's momentum. On the other hand, these particles, can be expected to have, in general, a low transverse momentum (see Eq. 49 below), i.e., leading particle production is a soft process. The transverse momentum transfer, moreover, can almost be taken to be unrelated to the energies of the primaries (or, indeed, the secondaries) and is, instead, at an intuitive level at least, related to the inverse of the 'size' of the interacting hadrons $\sim 1 / \mathrm{fm}$ (e.g., Perkins (1987)).

To calculate the leading neutron production rate in pp collisions with 'beam' protons of lab energy $\gtrsim 5 \times 10^{18} \mathrm{eV}$ we need to know

1. What the total (inelastic) pp cross-section is at such an energy.

2. What proportion of pp interactions at such energies result in the production of leading neutrons, i.e. the leading neutron multiplicity. We expect here a number less than unity that we could equally well describe as the charge exchange probability (in pp interactions).

3. What fraction of the incoming proton's energy any leading neutron will typically have (i.e., the quantity for the leading neutron that has traditionally been labeled the elasticity by cosmic ray investigators).

We discuss the empirical inputs to each of these three in turn below, before we go on to consider theory and modeling.

\subsection{Hadronic Cross-sections at High Energies: Experiment}

Unlike the cases presented by leading particle multiplicities and inelasticities, hadronic cross-sections have been measured (almost) up to the highest center-of-mass energies we are 
interested in $(\sqrt{s} \sim 50 \mathrm{TeV})$, though it should be noted that at the highest energies the pp cross-section is extracted - using Glauber theory (Glauber and Matthiae 1970) - from cosmic-ray data on proton- 'air' nucleus interactions (see, e.g., Block (3)). Moreover, between $\sim 10^{11} \mathrm{eV}$ and $10^{13} \mathrm{eV}$ the cross-section has not been directly measured. These two facts, however, do not allow for any great uncertainty in the cross-section: clear of the various resonances at a few $\mathrm{GeV}$, the growth of the total pp cross-section becomes smooth and slow in the measured region. Further, there is a strict bound, the Froissart-Martin bound (Froissart 1961; Martin 1965), on the asymptotic behaviour of the total cross-section, viz., for $s \rightarrow \infty$

$$
\sigma_{t o t} \leq C \ln ^{2} s
$$

From parametrization of the growth of the total pp cross-section between collider energies and UHE cosmic ray data, the Froissart bound may be saturated.

As noted in the body of the paper, we take the pp cross-section to be constant for beam energies between about $10^{10} \mathrm{eV}$ and $10^{12} \mathrm{eV}$ which, from from fig. 39.12 of Hagiwara et al. (2002) is quite reasonable. On the other hand, we do allow for the slow growth (Hagiwara 2002) of the cross-section between this energy regime and that relevant to the EHE neutron production.

\subsection{Leading Neutron Multiplicity in Proton-Proton Interactions: Experiment}

At the EeV energy regime we are interested in, there is no direct, empirical data on leading neutron production. Indeed, there are, in general, great difficulties in obtaining information on production of (even) charged leading particles from colliding beam devices (let alone neutrons). This is because the combination of large longitudinal and relatively low transverse momentum that, as mentioned above, characterizes leading particles means that they are kinematically constrained to emerge from their creation region at small angles. They will, therefore, be traveling down a collider's beam pipe where there is little or no detector acceptance. In fact, for $p p \rightarrow$ leading $n$, the most recent and highest energy data were obtained in the mid 1970s at experiments conducted at up to $\sqrt{s} \sim 63 \mathrm{GeV}$ at the ISR (Engler et al. 1975; Flauger and Mönning 1976) — three orders of magnitude below the energy regime we are interested in — and Fermilab (Dao et al. 1974). These data are described to first order by Feynman scaling (Feynman 1969) which reflects the partonic make-up of the colliding protons (see below). Also of relevance, data have been obtained for leading neutron production in proton-heavy ion $(p A)$ and heavy ion-heavy ion collisions (for recent data see, e.g. Barish et al. 2002, Huang et al. 2002, Armstrong et al. 1999) and for 
$e^{+} p$ collisions by the ZEUS Collaboration at Hera (Chekanov and al. 2002; Levman 2002b; Levman 2002a).

In the astrophysics literature a wide range of values for the leading neutron multiplicity has been employed from $\sim 0.1$ (Contopoulos and Kazanas 1995) to $\sim 0.5$ (Berezinskii \& Gazizov 1977) with values close to one quarter perhaps most generally being favored (e.g., Sikora (1989); Jones (1990); Drury (1994)). But, given that this preference seems a matter of lore, one must really look to the data.

Unfortunately, here again the picture is not entirely clear. From fits to the ISR data (Engler et al. 1975; Flauger and Mönning 1976), obtained at CMS energies between around 16 and $63 \mathrm{GeV}$, various researchers have determined values for the leading neutron multiplicity, $n_{n}$, between 0.25 and 0.40 . Though we have not re-analysed the data ourselves, our considered opinion is that a neutron multiplicity at the higher end of this range is actually the best motivated. In support of this position, note that, for instance, though (from the ISR data) Frichter (13) obtain $n_{n}=0.27$, they also find a value for the leading proton multiplicity, $n_{p}$, of 0.62 which, together with the constraint that $n_{p}+n_{n}=1$ (where leading hyperon production, as supported by the data, can be neglected approximately), is consistent with a value for the leading neutron multiplicity at the higher end of the above range (and, of course, the proton is generically easier to track than the neutron). Also note that from the same data, Forti et al. (1990) find $n_{n}=.40$. Further, from lower energy experiments conducted at the $2 \mathrm{~m}$ hydrogen bubble at the CERN proton synchrotron in the late 1970s, Blobel et al. (1978) determine $\sigma_{n} /\left(\sigma_{p}+\sigma_{n}\right)$ to be $\sim 0.38$ and 0.35 at CMS energies of 12 and $24 \mathrm{GeV}$ respectively (where $\sigma_{p}$ and $\sigma_{n}$ are, respectively, the total, inclusive cross-sections for $p p \rightarrow p p X$ and $p p \rightarrow p n X)$. As we set out below, the more sophisticated theoretical treatments also predict a leading neutron multiplicity closer to one half than one quarter. In conclusion, therefore, we take it that the value of $n_{n}$ is 0.40 at ISR energies.

\subsection{Leading Neutron Elasticity in Proton-Proton Interactions: Experiment}

Again, there is a complete lack of direct, leading particle inelasticty measurements at the EeV energy scales we are concerned with and we can only look to the ISR data. For the Feynman x variable

$$
x_{F} \equiv p_{\|} / p_{\|}^{\max } \simeq 2 p_{\|} / \sqrt{s},
$$

which, at the energies of interest we can take to be indentical to the inelasticity, Frichter (13) find from the ISR data average values for protons and neutrons of $\left\langle x_{F p}\right\rangle=0.44$ and $\left\langle x_{F n}\right\rangle=0.26$. The fact that the data indicate that leading neutrons be softer than leading protons is consistent with the expectation from theory: see below. 


\subsection{Theory at Low Energies and the Interpretation of the ISR Data}

If Feynman scaling were exactly obeyed, the inclusive distribution of final state particles would be given by:

$$
\begin{aligned}
\frac{d \sigma_{p p \rightarrow a}}{d p_{\|} d^{2} p_{\perp}}\left(p_{\|}, p_{\perp}, \sqrt{s}\right) & \simeq f_{a}\left(p_{\|}, \sqrt{s}\right) G_{a}\left(p_{\perp}\right) \\
& \simeq \frac{F_{a}\left(x_{F}\right)}{\sqrt{s}} G_{a}\left(p_{\perp}\right),
\end{aligned}
$$

where the functions $F_{a}\left(x_{F}\right)$ and

$$
G_{a}\left(p_{\perp}\right) \propto e^{-b p_{\perp}^{2}}
$$

are independent of $\sqrt{s}$ (Lipari 2003). The ISR data are, in fact, well described by such scaling: the cross-section for leading neutron production was found to fall off exponentially in $p_{\perp}$ for fixed Feynman $x_{F}$ and could be parameterized by equation (48) with $b$ parameter of $(4.8 \pm 0.3)(\mathrm{GeV} / c)^{-1}$, independent of $s$ and $x_{F}$ in the range $0.2 \geq x_{F} \geq 0.7$

(Engler et al. 1975). Further, the invariant cross-section shows no dependence on $s$ in the ISR data.

At the energies probed by this experiment, the dominant mechanism for leading neutron production in pp is direct production mediated by single pion exchange $\left(\pi^{+}\right)$. The $\pi$ dominates the ISR-energy $p \rightarrow n$ transition amplitude because of its low mass, its relative contribution increasing for $|t|$ decreasing (Chekanov and al. 2002). Other processes, which could otherwise be expected to contribute at the 20-30\% level - and which are required for the self-consistency of the meson exchange picture - in fact only contribute marginally $(5 \%$ level; Levman et al. 2002a). Such secondary processes include exchange of the $\rho$ and $a_{2}$ mesons, off mass-shell effects and absorption. There can also be marginal contributions from two-meson exchange, and different exchanges can interfere (Levman 2002b). Indirect production processes including diffractive (or resonant) production of heavy baryons also play a part (i.e., $\Delta^{+}$and $\Delta^{0}$ resonances; these two being excited baryon multiplets of uud and udd quarks, respectively).

As set out above it was demonstrated at the ISR that leading neutrons are significantly softer than their proton counterparts. This can be understood in the following way: the emergence of a leading neutron from a pp interaction requires that one of the incident protons has 'swapped' a valence $u$ for a valence $d$. But the $u$ that has been cast out may itself be hadronized into a leading meson which would be expected to carry a large fraction of the initial momentum. This suppresses leading neutron production at $x_{F} \rightarrow 1$. 


\subsection{Extrapolating Multiplicity and Elasticity to High Energies: Gribov-Regge Theory and Montecarlo Modeling}

Clearly if Feynman scaling always held exactly one could easily extrapolate low energy results to predict the properties of hadronic interactions at an arbitrarily high energy. Data obtained on pp interactions in general (conducted since the ISR experiments) at Sp $\bar{p} S$, Tevatron and RHIC, however, have shown that Feynman scaling is violated with, in particular, both the afore-mentioned, slow cross-section growth and some evolution of secondary, charged particle multiplicity evident in the data (Lipari 2003). This can be naturally interpretted as symptomatic of the fact that at increasingly high 'probe' momenta, the detailed QCD structure of the hadron is progressively revealed (i.e., the description of the proton as a bag of three valence quarks breaks down).

Unfortunately, the higher center-of-mass energy experiments mentioned above (which, admittedly, are still at center-of-mass energies below our region of concern) have not been able to probe $p p \rightarrow$ leading $n$ (for the general reasons outline above). Experimental data, then, on multiplicity and elasticity must be extrapolated over fully three orders of magnitude (required to reach the super-EeV scales that concern us) using strong interaction models and this extrapolation may not assume Feynman scaling. But here, again, we run into problems because the leading particle production processes are soft and, therefore, associated with long distances. They cannot, then, be studied with perturbative QCD. Instead we must rely on Regge theory and the instantiation of concepts like the Pomeron in Monte Carlo codes (see below).

A general framework to effect the extrapolation of hadronic interactions from collider energies to the super-EeV energy scales we are interested in has been realized over the last decade (Werner 1993; Engel 2001; Ostapchenko 2003) in a number of different Montecarlo codes. These are based on Gribov's Reggeon Field Theory or the QCD eikonal approach (Ostapchenko 2003). In both these, a single hadronic interaction can be broken down into a set of pomeron exchanges between participating partons (Gribov 1968; Engel 2001; Lipari 2003) ${ }^{14}$. As explained above, the increasing number of parton-parton interactions that open up with increasing $\sqrt{s}$ explain the weak energy dependence (and, therefore Feynman-scaling violation) seen in the cross-section, multiplicity, etc., data. Further, an approach involving muliple particle scattering, seems to be the requirement if one is to arrive at a self-consistent

\footnotetext{
${ }^{14}$ Note that while single-pomeron exchange generally predict results indistinguishable from these models at energies up to several $\mathrm{TeV}$ in the center-of-mass, the single-pomeron exchange amplitude eventually violates unitarity. Multiple pomeron exchange, however, predicts a weaker energy growth above this energy scale and cures the unitarity problem: (11).
} 
description of Feynman scaling violations in secondary particle spectra (Ostapchenko 2003). This topic is beyond the scope of this work; we simply quote results obtained by other researchers below.

\subsubsection{Leading Neutron Multiplicity at High Energies}

In modeling of non-diffractive production, the ratio of leading proton to leading neutron reflects the proton fragmentation scheme employed (Huang 2002). Again, if scaling were exactly obeyed this ratio would show no dependence on $\sqrt{s}$. More complicated models predict a small energy dependence in this ratio ${ }^{15}$ (this is also the case for diffractive production). Such energy dependence as does arise is due to the increase in the number of sub-interactions between partons that occurs for increasing $\sqrt{s}$ (Lipari 2003). In a simple diquark-quark proton fragmentation scheme, the incident proton can fragment into either a $u u$ or a $u d$ diquark. The uu must hadronize into a proton, but the ud can fragment into either a proton or a neutron. Thus, this simple scheme predicts a proton-to-neutron ratio of 2:1 for leading baryons (so $n_{n}$ would be $\sim 0.33$ ). More sophisticated schemes-like three-quark fragmentation and gluon-junction interaction models - predict, however, ratios closer to 1:1 (Huang 2002).

\subsubsection{Leading Neutron Elasticity at High Energies}

In regard to the simulation of UHE particle production with Monte Carlo codes, particular regard has been paid to leading neutron production by Capdevielle, Attallah and coworkers (Capdevielle, Attallah, \& Gabinski 1992; Capdevielle \& Attallah 1992). Employing a Monte Carlo generator developed for the simulation of cosmic ray cascades on the basis of a simplified picture of the dual parton model (Capella et al. 1994), these authors have investigated neutron production up to the centre of mass energy regime of $\sqrt{s}=50 \mathrm{TeV}$ that we are interested in. Their results incorporate direct production of leading neutrons and diffractive production through the $\Delta^{+}$and $\Delta^{0}$. In addition to the leading neutrons, they simulate production of neutron secondaries with small rapidity in nucleon-anti-nucleon pairs and through heavy baryon production and decay ( $\Lambda$ 's, $\Xi$ 's and $\Sigma$ 's: (Capdevielle \& Attallah 1992)). On the basis of an extrapolation of UA5 data, Capdevielle and Attallah determine that the average energy carried away by a leading neutron is $23-24 \%$ that of the incoming energy. This figure is very close to the neutron elasticity obtained from the ISR data, and, therefore

\footnotetext{
${ }^{15}$ Paolo Lipari 2003, private communication.
} 
consistent with (at worst) a weak energy-dependence that we may disregard.

\subsection{Conclusion: Final Assumptions on Leading Neutron Multiplicty and Inelasticity at EHE}

In summary, we have the following:

1. Data indicate that the total (inelastic) cross-section for pp interactions grows slowly with $\sqrt{s}$ so that scaling is violated. We account for this effect in this paper.

2. The highest energy experimental data available indicate, for pp interactions, a leading neutron multiplicity expectation of $n_{n}=0.4$ and an averaged leading neutron elasticity of $\left\langle x_{n}\right\rangle=0.25$.

3. Modeling indicates that $n_{n}$ and $\left\langle x_{n}\right\rangle$ are, at worst, only weakly dependent on CMS energy. As our final position, then, we adopt the above values for our calculations and take them to hold independent of $s$.

\section{2. $\chi^{2}$ Fitting Function}

For the sake of completeness, we set out the $\chi^{2}$ functions we have defined to provide a quantitative estimate of the goodness-of-fit of our model of the totality of the data (i.e., EGRET, HESS and EHE cosmic ray measurements). For particular instances described in the body of the paper we define an overall $\chi^{2}$ with contributions, as appropriate, from elements of

$$
\left\{\chi_{\text {EGRET }}^{2}, \chi_{\text {HESS }}^{2}, \chi_{\text {Neutron }}^{2}\right\} \text {. }
$$

The total $\chi^{2}$ is minimized by fitting to the spectral index and to the natural logarithm of the normalization of the $\gamma$-ray differential flux which is assumed to be governed by a power law. We work in logarithms because this means that, to a good approximation, the fitted quantities are linear in the fitting parameters. We use the result that, for a linear model $y(x)=a+b x$, in which the experimental limitations mean that both the observed quantities $\left(y_{i}^{\prime} s\right)$ and the parameter values at which they are observed $\left(x_{i}^{\prime} s\right)$ are uncertain, a $\chi^{2}$ may be defined by

$$
\chi^{2}=\sum_{i} \frac{\left(y_{i}-a-b x_{i}\right)^{2}}{\sigma_{y_{i}}^{2}+b^{2} \sigma_{x_{i}}^{2}} .
$$

We find, then, (we disregard the units of naturally dimensionful quantities and take all energies to be measured in $\mathrm{eV}$, all fluxes in $\mathrm{cm}^{-2} \mathrm{~s}^{-1}$, and all differential fluxes in $\mathrm{cm}^{-2} \mathrm{~s}^{-1}$ 
$\left.\mathrm{eV}^{-1}\right)$

$$
\chi_{\mathrm{EGRET}}^{2}=\sum_{i} \frac{\left(\log \left[\frac{d F_{\mathrm{EGRET}}^{o b s}\left(E_{i}\right)}{d E}\right]-\log [N \text { orm }]+\gamma \log \left[E_{i}\right]\right)^{2}}{\left(\Delta\left[\log \left[\frac{d F_{\mathrm{EGRET}}^{o o b s}\left(E_{i}\right)}{d E}\right]\right]\right)^{2}+\gamma^{2}\left(\Delta\left[\log \left[E_{i}\right]\right]\right)^{2}}
$$

and

$$
\chi_{\text {Neutron }}^{2}=\frac{\left(\log \left[F_{n}^{o b s}\right]-\log \left[F_{n}^{\text {pred }}(\gamma, \text { norm })\right]\right)^{2}}{\left(\Delta\left[\log \left[F_{n}^{o b s}\right]\right]\right)^{2}+(1-\gamma)^{2}\left(\Delta\left[\log \left[E_{\text {Neutron }}\right]\right]\right)^{2}} .
$$

The HESS $\chi^{2}$ has the same form as the EGRET $\chi^{2}$. There are a number of points to note in relation to these equations. Firstly, Norm - a number in $\mathrm{cm}^{-2} \mathrm{~s}^{-1} \mathrm{eV}^{-1}-$ denotes the fitted, $\gamma$-ray, differential flux normalization. Secondly, $F_{n}^{\text {pred }}$ in the last equation is as given by Equations (23) and (24). Thirdly, strictly the $\chi^{2}$ analysis only holds for the case of Gaussian statistics in which case errors should be symmetric about the measured values (of the logarithms of the energies or fluxes). Our data do not always precisely satisfy this criterion. In the cases where they do not, we take, then, the geometrical mean of the ratios (best value):(smallest value) and (largest value):(best value) to define the error. Lastly, the sum in the EGRET $\chi^{2}$ is over the last three data points obtained from this instrument's $\mathrm{GC}$ observation (this is to guarantee that we are in the scaling regime so that there be no curvature in the photon differential flux).

\section{Derivation of the Maximum Energy}

In this appendix we present the derivation of equation (39). From Eqs. (31) and (35), we have for a perpendicular shock

$$
\begin{aligned}
\frac{1}{p_{c}} \frac{\mathrm{d} p_{c}}{\mathrm{~d} t} & \simeq \frac{U_{\text {shock }}^{2}}{4 \kappa_{\perp}} \\
& =\frac{U_{\text {shock }}^{2}}{4 \kappa_{\|}}\left[1+\left(\frac{\lambda_{\|}}{r_{g}}\right)^{2}\right] \\
& =\frac{3}{4 \lambda_{\|}} \frac{U_{\text {shock }}^{2}}{w}\left[1+\left(\frac{\lambda_{\|}}{r_{g}}\right)^{2}\right] \\
& \simeq \frac{3}{4} \frac{U_{\text {shock }}^{2} \lambda_{\|}}{w r_{c}^{2}}
\end{aligned}
$$

We again take the maximum value of $\Upsilon \sim 0.3$, so that

$$
\frac{U_{\text {shock }}}{w} \frac{\lambda_{\|}}{r_{c}} \rightarrow 0.3
$$


so that substituting equation (55) into equation (54) we find

$$
\frac{1}{p_{c}} \frac{\mathrm{d} p_{c}}{\mathrm{~d} t} \simeq \frac{1}{4} \frac{U_{\mathrm{shock}}}{r_{c}}
$$

Noting that the gyroradius is

$$
r_{g}=\frac{p_{\perp}}{e B}
$$

we have that $r_{c} \simeq p_{c} / e B$. Therefore,

$$
p_{c} \simeq \frac{e B}{4} \int U_{\text {shock }} d t
$$

or to put this in a more observationally-motivated form,

$$
p_{c} \simeq \frac{e B}{4} R_{\text {shock }}
$$

where $R_{\text {shock }}$ is the characteristic shock radius. Numerically,

$$
p_{c} \simeq 5 \times 10^{16} Z\left(\frac{B}{20 \mu G}\right)\left(\frac{R_{\text {shock }}}{10 \mathrm{pc}}\right) \frac{\mathrm{eV}}{c} .
$$

\section{REFERENCES}

Aharonian, F. A. et al. 2004, pre-print(astro-ph/0408145). Submitted to A\&A.

Aharonian, F. A. et al. 2002, A\&A, 395, 803

Alvarez-Muñiz, J. and Halzen, F. 2002, ApJL, 576, L33

Baring, M. G. et al. 1999, ApJ, 513, 311

Bednarek, W., Giller, M., and Zielinska, M. 2002, Journal of Physics G Nuclear Physics, 28, 2283

Begelman, M. C., Rudak, B., and Sikora, M. 1990, ApJ, 362, 38

Bellido, J. A., Clay, R. W., Dawson, B. R., and Johnston-Hollitt, M. 2001, Astroparticle Physics, 15, 167

Berezinskii, V. S. and Gazizov, A. Z. 1977, Journal of Experimental and Theoretical Physics Letteres, 25, 254

Biermann, P. L. et al. 1999, ApJ, 604, L29 
Bird, D. J. et al. 1999, ApJ, 511, 739

Blasi, P. and Melia, F. 2002, submitted to MNRAS

Blobel, V., Fesefeldt, H., Franz, H., Geist, W. M., von Holt, K., Idschok, U., and Schmitz, N. 1978, Nuclear Physics B, 135, 379

Block, M. M., Halzen, F., and Stanev, T. 2000, Phys. Rev. D, 62, 77501

Bossa, M., Mollerach, S., and Roulet, E. 2003, Journal of Physics G Nuclear Physics, 29, 1409

Camin, D. V. 2004, Nuclear Instruments and Methods in Physics Research A, 518, 172

Candia, J., Epele, L. N., and Roulet, E. 2002, Astroparticle Physics, 17, 23-33.

Candia, J., Mollerach, S., and Roulet, E. 2002, Journal of High Energy Physics, 12, 32

Capdevielle, J. N. and Attallah, R. 1992. Limitation of leading particle effect by vector meson and resonances produced in EAS. In Very High Energy Cosmic Ray Interactions, page 448. Given at 7th International Symposium on Very High-energy Cosmic Ray Interactions, Ann Arbor, MI, 21-27 Jun 1992

Capdevielle, J. N., Attallah, R., and Gabinski, P. 1992. Introduction to neutron astronomy. In Very High Energy Cosmic Ray Interactions, page 442. Given at 7th International Symposium on Very High-energy Cosmic Ray Interactions, Ann Arbor, MI, 21-27 Jun 1992

Capella, A., Sukhatme, U., Tan, C.-I., and Tran Thanh Van, J. 1994, Phys. Rept., 236, $225-329$

Carraminana, A. 1992, A\&A, 264, 127

Chekanov, S. and al. 2002, Nuclear Physics B, 637, 3

Chilingarian, A., Martirosian, H., and Gharagyozyan, G. 2003, ApJl, 597, L129

Clay, R. W., Dawson, B. R., Bowen, J., and Debes, M. 2000, Astropart. Phys., 12, 249

Cline, D. B. 1988 . In Augenstein, B., editor, Proceedings of the RAND Workshop on Antiproton Science and Technology, page 45. (New Jersey: World Scientific)

Contopoulos, J. and Kazanas, D. 1995, ApJ, 441, 521

Crocker, R. M., Melia, F., and Volkas, R. R. 2000, ApJS, 130, 339 
Crocker, R. M., Melia, F., and Volkas, R. R. 2002, ApJS, 141, 147

Dao, F. T. et al. 1974, Phys. Rev. D, 10, 3588

Dazeley, S. A., Patterson, J. R., Rowell, G. P., and Edwards, P. G. 2001, Astroparticle Physics, 15, 313

Dermer, C. D. 2002, ApJ, 574, 65

Di Cocco, G. et al. 2004, pre-print(astro-ph/0403676)

Drury, L. O., Aharonian, F. A., and Voelk, H. J. 1994, A\&A, 287, 959

Eckart, A. and Genzel, R. 1997, MNRAS, 284, 576

Engel, R. 2001, pre-print(hep-ph/0111396)

Engel, R., Seckel, D., and Stanev, T. 2001, Phys. Rev. D, 64, 93010

Engler, J. et al. 1975, Nuclear Physics B, 84, 70

Fatuzzo, M., Melia, F., and Rafelski, J. 2001, ApJ, 549, 293

Fatuzzo, M. and Melia, F. 2003, ApJ, 596, 1035

Feynman, R. P. 1969, Physical Review Letters, 23, 1415

Flauger, W. and Mönning, F. 1976, Nuclear Physics B, 109, 347.

Fleysher, R. and Milagro Collaboration 2002, Bulletin of the American Astronomical Society, 34,676

Forti, C., Bilokon, H., D’Ettorre Piazzoli, B., Gaisser, T. K., Satta, L., and Stanev, T. 1990, Phys. Rev. D, 42, 3668

Frichter, G. M., Gaisser, T. K., and Stanev, T. 1997, Phys. Rev. D, 56, 3135

Froissart, M. 1961, Physical Review, 123, 1053

Gaisser, T. K. 1990, Cosmic rays and particle physics, Cambridge, UK: Cambridge University Press

Genzel, R. et al. 2003, Nature, 425, 934

Ghez, A. M. et al. 2003, ApJl, 586, L127 
Glauber, R. J. and Matthiae, G. 1970, Nuclear Physics B, 21, 135

Gribov, V. N. 1968, Sov. Phys. JETP, 26, 414

Hagiwara, K. et al. 2002, Phys. Rev. D, 66, 010001

Hartman, R. C. et al. 1999, ApJS, 123, 79

Hayashida et al. 1999a, pre-print(astro-ph/9906056)

Hayashida, N. et al. 1999b, Astroparticle Physics, 10, 303

Hillas, A. M. 1980, Proc. 16th Int. Cosmic Ray Conf. (Kyoto), 6, 13

Hinton, J. A. 2004, New Astronomy Review, 48, 331

Hooper, D., et al. 2004, pre-print(astro-ph/0404205)

Hooper, D., and Dingus, B 2002, pre-print(astro-ph/0212509)

Huang, H. Z. 2002, J. Phys., G28, 1667

Jokipii, J. R. 1982, ApJ, 255, 716

Jokipii, J. R. 1987, ApJ, 313, 842

Jones, L. 1990. Direct Neutrons of over 0.5 EeV from Cygnus X-3. In Protheroe, R., editor, Proceedings of the 21st ICRC (Adelaide), volume 2, pages 75

Kosack, K. and Collaboration, t. V. 2004, pre-print(astro-ph/0403422)

Krennrich, F. et al. 2004, New Astronomy Review, 48, 345-349

Lagage, P. O. and Cesarsky, C. J. 1983, A\&A, 125, 249

Levman, G. 2002a, Journal of Physics G Nuclear Physics, 28, 1079

Levman, G. 2002b, Nuclear Physics B, 642, 3

Lipari, P. 2003, Nucl. Phys. Proc. Suppl., 122, 133

Longair, M. S. 1994, High energy astrophysics. Vol.2: Stars, the galaxy and the interstellar medium. Cambridge: Cambridge University Press, —c1994, 2nd ed.

Markoff, S., Melia, F., and Sarcevic, I. 1997, ApJ, 489, L47

Markoff, S., Melia, F., and Sarcevic, I. 1999, ApJ, 522, 870 
Martin, A. 1965, Nuovo Cim., A42, 930

Mayer-Hasselwander, H. et al. 1998, A\&A, 335, 335

Melia, F. and Falcke, H. 2001, ARA\&A, 39,309

Melia, F., Fatuzzo, M., Yusef-Zadeh, F., and Markoff, S. 1998, ApJL, 508, L65

Moskalenko, I. V. 1995, Space Science Reviews, 72, 593

Mucke, A. et al. 1999, PASA, 16, 160

Ostapchenko, S. S. 2003, Journal of Physics G Nuclear Physics, 29, 831

Parker, E. N. 1965, Planetary and Space Science, 13, 9

Pedlar, A., Anantharamaiah, K. R., Ekers, R. D., Goss, W. M., van Gorkom, J. H., Schwarz, U. J., and Zhao, J. 1989, ApJ, 342, 769

Perkins, D. H. 1987. Introduction to High-Energy Physics, Third Ed. Reading, USA: AddisonWesley

Shklovskii, I. S. 1953, Dokl. Akad. Nauk SSSR, 91, 475

Sikora, M., Begelman, M. C., and Rudak, B. 1989, ApJl, 341, L33

Stecker, F. W. 1979, ApJ, 228, 919

Telesco, C. M. and Davidson, J. A. and Werner, M. W. 1989, ApJ, 456, 541

Tkaczyk, W. 1989, ApJ, 92, S611

Takahashi, K. and Nagataki, S. 2001, pre-print(astro-ph/0108507)

Takeda, M. 1999. Prepared for 26th International Cosmic Ray Conference (ICRC 99), Salt Lake City, Utah, 17-25 Aug 1999

Thompson, D. J. 2004, New Astronomy Review, 48, 543

Tsuchiya, K. et al. 2004, ApJ, 606, L115

Werner, K. 1993, Phys. Rept., 232, 87

Wolfire, M. G. and Tielens, A. G. G. M. and Hollenbach, D. 1990, ApJ, 358, 116 


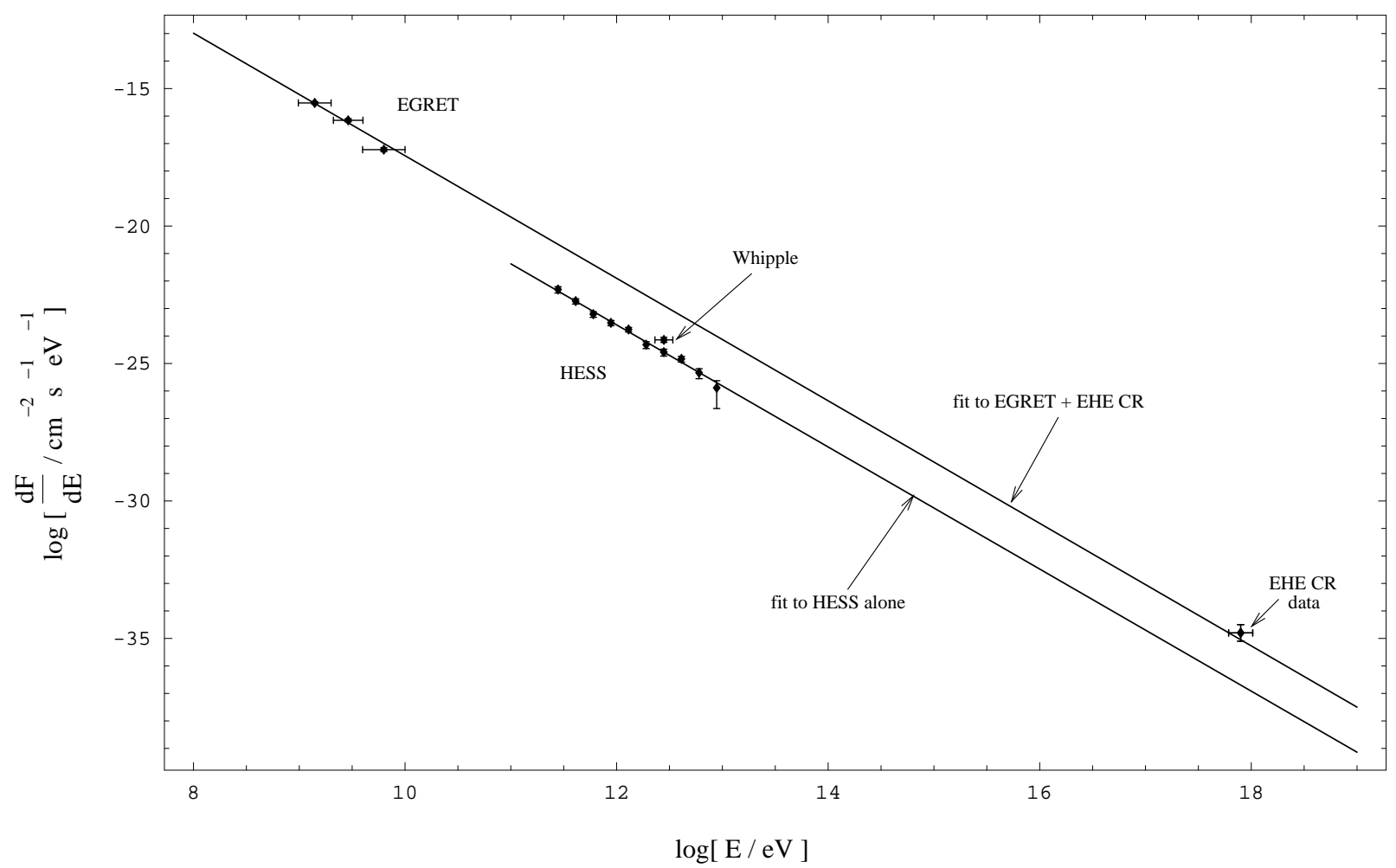

Fig. 1. $-\gamma$-ray and neutron differential fluxes together with fitted curves. The three points on the left of the figure are from EGRET (Mayer-Hasselwander et al. 1998). The 11 data points in the middle of the figure are GC, $\gamma$-ray differential fluxes measured by atmospheric cerenkov telescopes. Amongst these, the single point with marked error bars sitting proud of the fitted line is due to Whipple (Kosack et al. 2004), the other 10 points are from the recent HESS July/August 2003 data set (Aharonian et al. 2004). The right data point gives the neutron flux which, on the basis of the EHE cosmic ray data, we have taken to be $1.0_{-0.5}^{+1.0} \times 10^{-17} \mathrm{~cm}^{-2} \mathrm{~s}^{-1}$ above $10^{17.9} \mathrm{eV}$. The upper line gives the best-fit (as described in the text) photon differential flux obtained from a simulataneous fit to the EGRET and EHE cosmic ray data. This is given by a power law with a spectral index of 2.23 (the curve would be inaccurate at EHE because it does not take into account the growth of the total pp cross-section). Obscured by (i.e., in excellent agreement with) the right data point is a triangle indicating the position of the neutron differential flux at $10^{17.9} \mathrm{eV}$ as determined by the best-fit power law (that this point is apparently on top of the $\gamma$-ray flux curve is coincidental). The lower curve - with a spectral index of 2.22 - has been found by fitting a power law to the HESS data alone. Note how extremely closely the spectral indices match. 


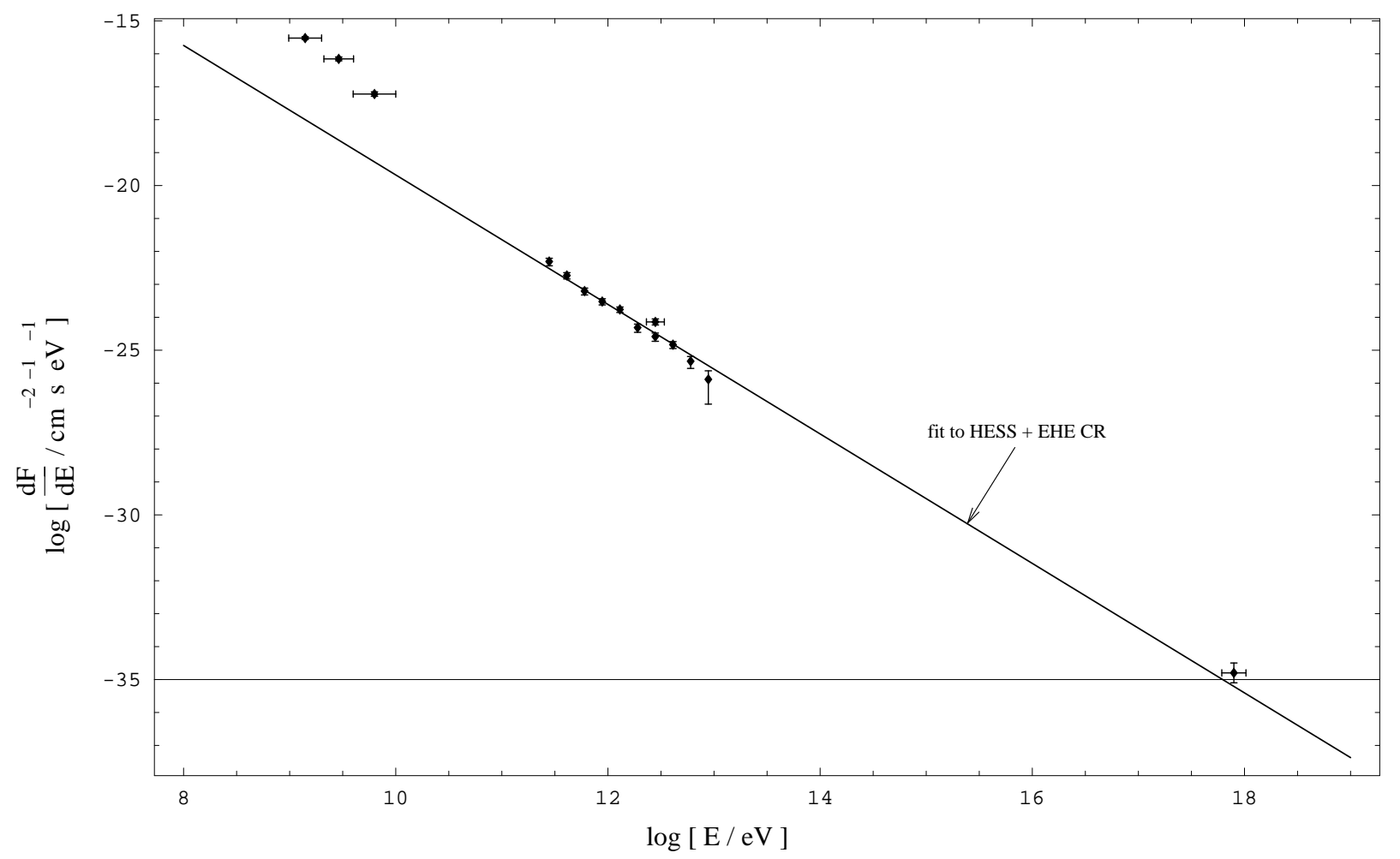

Fig. 2.- $-\gamma$-ray and neutron differential fluxes together with another fitted curve. The data are as given in the previous figure. The curve is obtained from a power law fit (simulataneously) to the HESS $\sim \mathrm{TeV} \gamma$-ray data (but not the Whipple data point) and the EHE cosmic ray data (again, note that the curve would be inaccurate at EHE because it does not take into account the growth of the total pp cross-section). The best-fit spectral index is 1.97. The EGRET data points have not been used in this fit. On the right is illustrated both the EHE CR data point and (again obscured by the former) a triangle indicating the position of the neutron differential flux at $10^{17.9} \mathrm{eV}$ as determined by the best-fit power law. 